\title{
THE DELPHI APPROACH TO MACRO MODEL ESTIMATION
}

\author{
A.J. Berkhout, Henk Cox, Eric Verschuur and Kees Wapenaar \\ Delft University of Technology \\ Laboratory of Seismics and Acoustics \\ PO Box 5046 \\ 2600 GA Delft \\ The Netherlands
}

\section{ABSTRACT}

At first glance it may appear attractive to aim at one large inversion scheme that transforms seismic measurements into rock and pore parameters. However, apart from the gigantic computation times involved, the development of one inversion scheme may not be the best way to go!

Experience confirms that if the total inversion is carried out in one step, the problem is seriously illposed and difficult to stabilize in a meaningful way. However, if the inversion is carried out in consecutive steps, each step can be stabilized by available prior information and the result of each step can be critically evaluated before the next step is started (maximum user control).

Full seismic inversion, i.e. transforming seismic shot records into geologically-oriented subsurface parameters, is carried out in 'Delphi' by six consecutive steps, realized in three separate acoustic (marine) or elastic (land) processing modules. In the first module the surface information, i.e. the source input signal and the detector output signals, are transformed into respectively the downgoing source wave field and the upgoing reflected wave fields at the surface. Next, the influence of the source wavelet and the surface reflectivity are removed from the wave fields by a novel data adaptive multiple elimination method. In the second module the macro subsurface model is estimated and, next, the source wave fields and reflected wave fields are extrapolated from the surface into the subsurface; for each subsurface grid point (depth point) the angle dependent reflectivity property is computed. Finally, in the third module the reflectivity information is transformed into velocity and density maps and, next, into rock and pore parameters. The last step is only feasible if a significant amount of nonseismic information is available. This means that, particulary in the last inversion module, an integrated approach is required.

This chapter starts with an outline of the Delphi scheme (Delphi is a consortium project at the Laboratory of Seismics and Acoustics in Delft). Furthermore, we discuss the processing of the 'Marmousi data' according to the Delphi approach. 

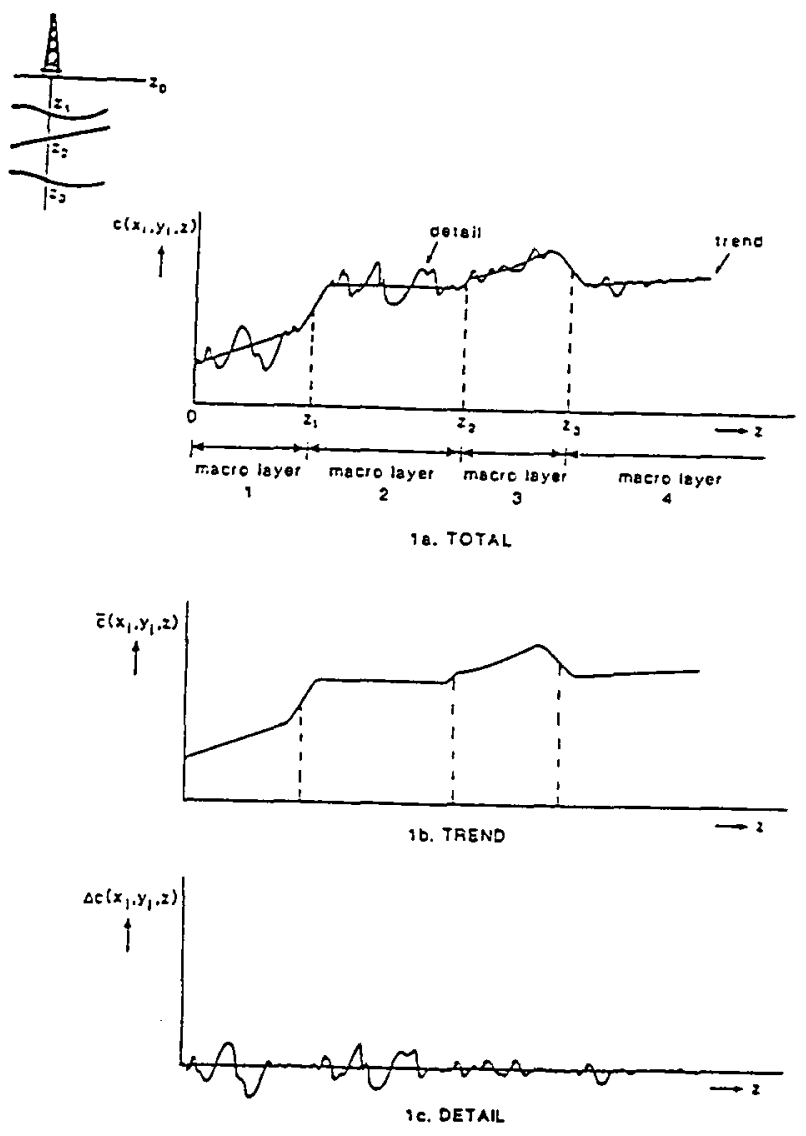

Figure I

Description of the subsurface in terms of trend and detail

essence of

seismic method

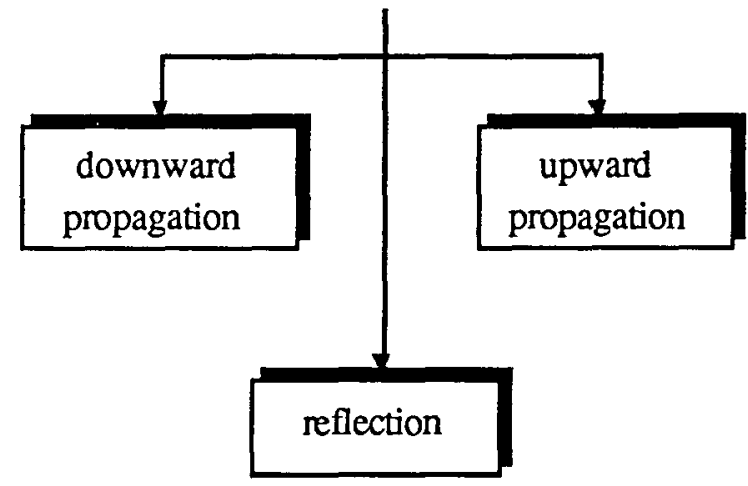

Figure 2

Seismic responses are defined by the propagation and reflection properties of the subsurface.

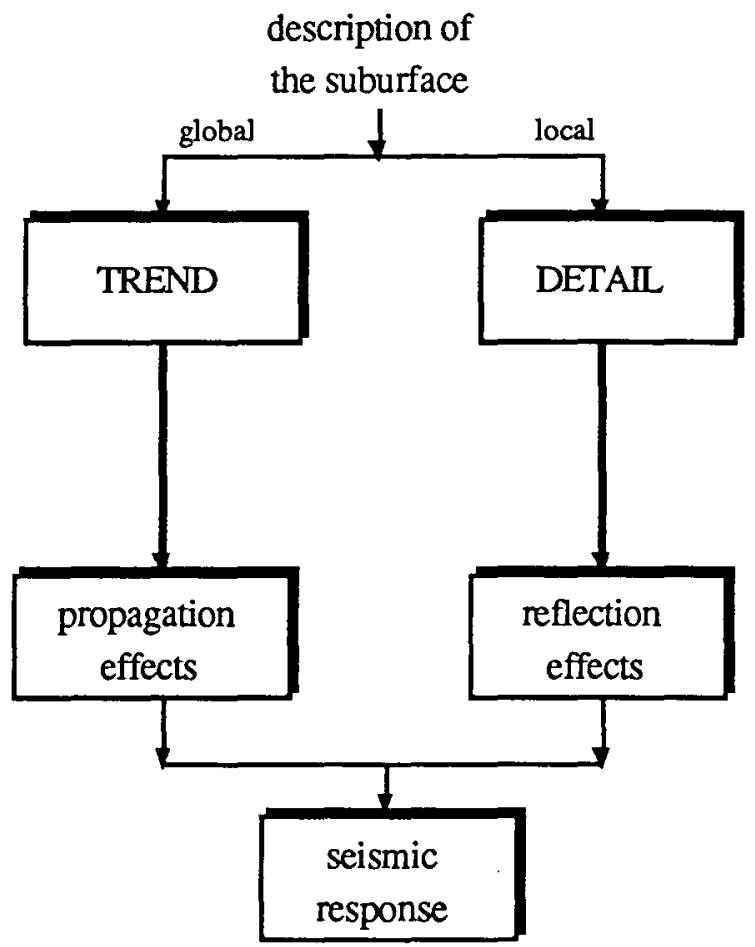

Figure 3

Trend and detail in the subsurface determine the propagation and reflection effects in the seismic response respectively. 


\section{OUTLINE OF THE DELPHI APPROACH}

\section{Introduction}

In conventional seismic processing techniques the measured seismic wave fields at the surface are processed in the time domain (deconvolution, CMP stacking, time migration). Therefore, conventional seismic processing may be referred to as timeoriented. In the modern view of seismic processing it is realized that accurate information on the subsurface (structural, stratigraphic, lithologic) can only be obtained if the wave fields, measured at the surface, are downward extrapolated to the subsurface grid points (depth points) of interest. Therefore, modern seismic processing may be referred to as depth-oriented. It may be stated that the conventional time domain approach provides an economic pre-view of the subsurface. However, if at selected areas a more accurate image is required then a depth point oriented approach is a prerequisite. Three dimensional, depth point oriented, acoustic and elastic seismic processing is the subject of the Delphi consortium.

\section{Description of the subsurface}

Figure 1a is a typical velocity profile obtained from well measurements. An interesting and important analysis of the measurement curve (velocity $\log$ ) is given by a subdivision in trend and detail. The trend (Figure $1 b$ ) gives information on the depth dependent compaction properties of the subsurface. The detail (Figure 1c) gives information on the different rock and pore properties of the individual geologic layers (within the resolution of the velocity $\log$ ). Using trend information, the subsurface may be subdivided in so-called macro layers, where each macro layer is characterized by its own trend parameters. One macro layer can be seen as a package of geologically related layers with the same compaction property. The distinction between trend and detail, or macro layering and fine layering, plays an important role in seismic inversion.

The essence of the seismic method is given by propagation and reflection (Figure 2). The source wave field propagates down in the subsurface, reflects at the layer boundaries and the reflected wave fields propagate back to the surface. Hence, the seismic response we measure at the surface represents a mixture of propagation and reflection information. The major part of seismic processing is dedicated to eliminate propagation effects from the seismic response, aiming at correctly positioned true amplitude reflectivity (numerical image formation). Going back to the afore mentioned description of the subsurface, we may make the important statement (Figure 3 ) that propagation is primarily determined by the relatively slow varying trend of the subsurface and reflection is primarily determined by the relatively fast varying detail of the subsurface. As a consequence, for the elimination of propagation effects the macro model of the subsurface should be available. Or in other words, seismic processing should be based on macro subsurface models. This means that estimation of macro models should be a key issue in the seismic industry.

\section{Description of seismic data}

The primary information at the surface $\left(\mathrm{z}=\mathrm{z}_{0}\right)$ from an inhomogeneous sub-surface can be elegantly presented by a matrix expression which may be found in chapter VI of Berkhout (1985). Here we treat the subject by illustration. The top of Figure 4 illustrates seismic propagation and reflection for one point source at the surface $\left(\mathrm{z}_{0}\right)$ and one reflection boundary $\left(\mathrm{z}_{\mathrm{m}}\right)$. Mathematically, the operators $\mathrm{W}^{+}$(the downward propagation operator from $z_{0}$ to $\mathrm{z}_{\mathrm{m}}$ ), $\mathbf{R}^{+}$(the reflection operator at the depth level $z_{m}$ for downward traveling waves) and $\mathbf{W}^{-}$(the upward propagation operator from $z_{m}$ to $z_{0}$ ) represent matrices, taking into account any type of vertical and lateral changes in the subsurface. The combination (see the bottom of Figure 4) of these operators and the source wave fields at the surface, represented by $\mathbf{S}^{+}\left(\mathrm{z}_{0}\right)$, yield the well-known data matrix $\mathbf{P}^{-}\left(z_{0}\right)$ containing all shot records. 

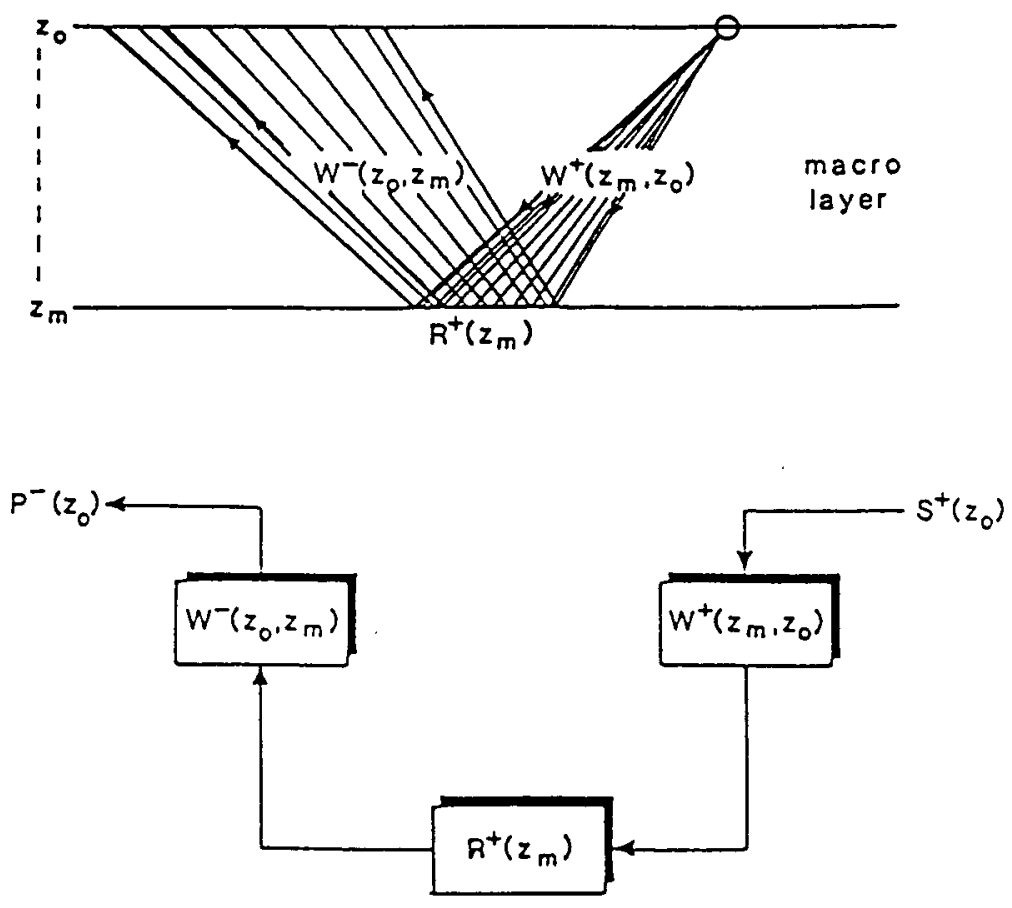

Figure 4

Propagation and reflection for one point source and one reflecting boundary, ignoring the reflectivity of the surface.
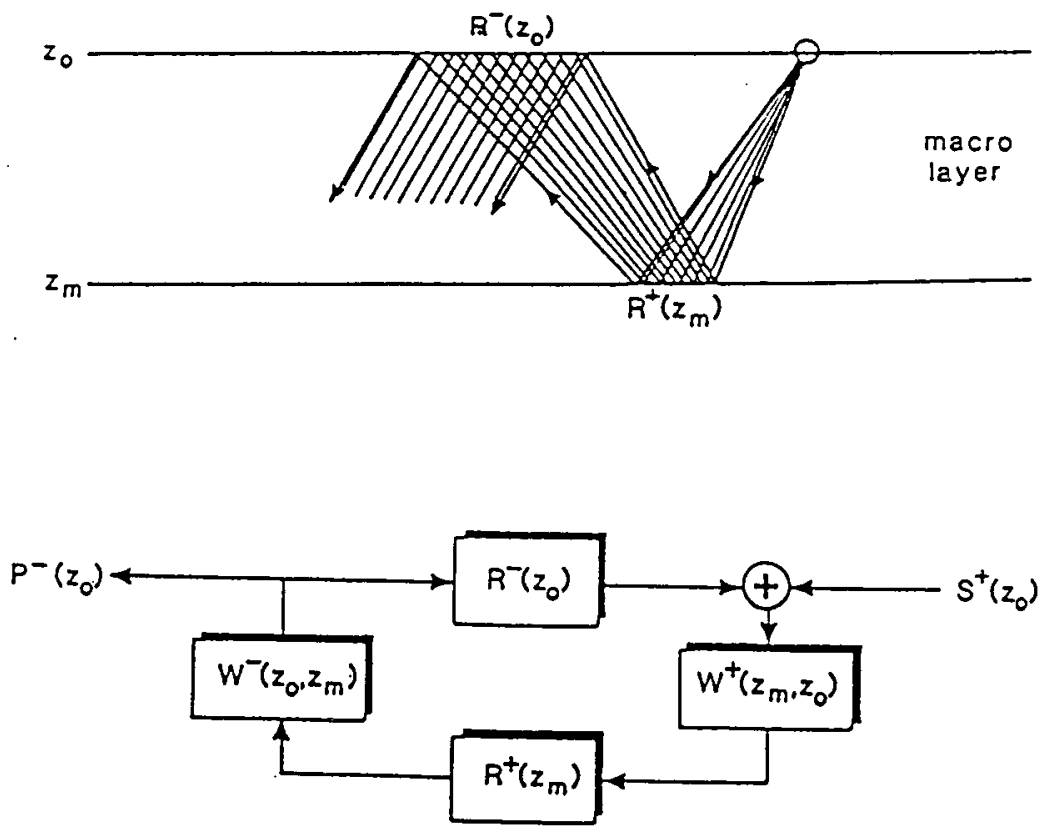

Figure 5

Propagation and reflection for one point source and one reflecting boundary, taking the reflectivity of the surface into account. 


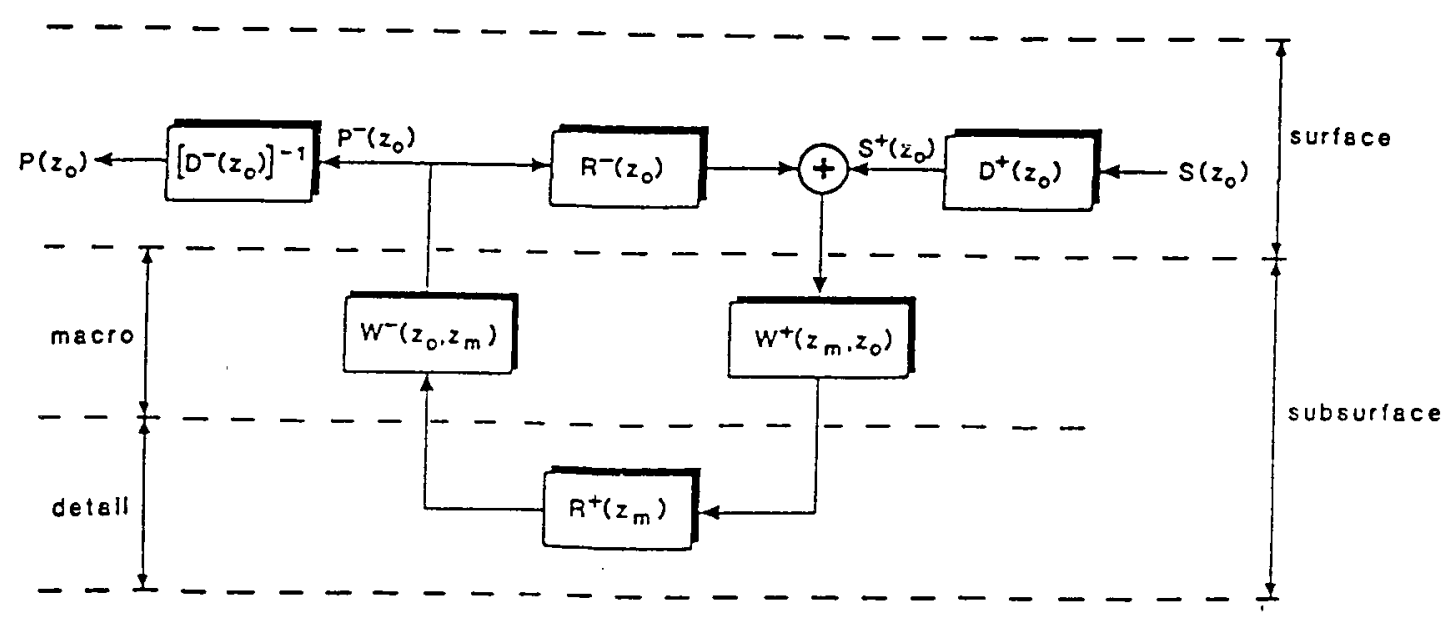

\section{Figure 6}

Summary on emission (at the surface), propagation, reflection (one reflecting boundary) and detection (at the surface).

In practice the surface $\left(z=z_{0}\right)$ is a strongly reflecting boundary and the model has to be modified to include a reflection operator at the surface for upward traveling waves, $\mathbf{R}^{-}\left(\mathrm{z}_{0}\right)$.

Figure 5 is a graphic representation for one point source and one reflection boundary in the subsurface. Note that in this case data matrix $\mathbf{P}^{-}\left(\mathrm{z}_{0}\right)$ contains all surface-related multiples. From simulations it can be easily demonstrated that in practical situations the multiple problem is largely caused by surface-related multiples.

Propagation, reflection and detection are summarized in Figure 6 . The decomposition operator $\mathbf{D}^{+}\left(\mathrm{z}_{0}\right)$ transforms the source function $S\left(z_{0}\right)$ into the downward travelling source wave field $\mathrm{S}^{+}\left(\mathrm{z}_{0}\right)$; at the other end the decomposition operator $\mathrm{D}^{-}\left(\mathrm{z}_{0}\right)$ transforms the measurements $\mathbf{P}\left(\mathrm{z}_{0}\right)$ to the upward travelling reflected wave fields $\mathbf{P}^{-}\left(\mathrm{z}_{0}\right)$.

If the mathematics is formulated in a recursive way then source(s) and detectors can be positioned anywhere in the subsurface and the internal multiples in propagation operators $\mathrm{W}^{+}\left(\mathrm{z}_{\mathrm{m}}, \mathrm{z}_{0}\right)$ and $\mathbf{W}^{-}\left(\mathrm{z}_{0}, \mathrm{z}_{\mathrm{m}}\right)$ can be easily quantified. Finally, note that the direct wave has been deleted.

\section{Inversion in steps}

As mentioned before, at first glance it may appear attractive to aim at one large inversion scheme that transforms seismic measurements into rock and pore parameters. However, apart from the gigantic computation times being involved, the development of one large inversion scheme may not be the best way to go. Figure 7 shows a stepwise inversion scheme, realized by three layers of software:

1. Surface-related pre-processing

2. Reflectivity imaging

3. Target-related post processing.

The essence of the stepwise approach is that, before leaving one software layer, consistency checks should be made to approve on the result. Those are the places where the interactive part of seismic processing pre-eminently fits. The processing in software layer 1 is largely based on discrete signal theory. Input as well as output are time sections. No specific information on the subsurface is required. Surface-related pre-processing should start with decomposition of the measured data into down- and upgoing waves. The suppression of surface waves and the elimination of surface-related multiples belong to software layer 1 as well. Note that conventional seismic processing (deconvolution, CMP stacking, time migration) should also be considered as a surface-related 
method, providing a pre-view of the subsurface.

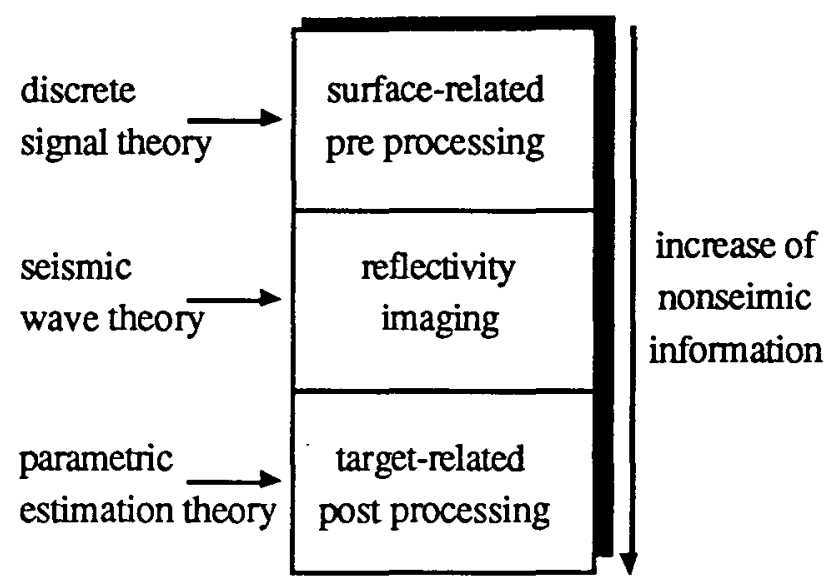

\section{Figure 7}

Stepwise seismic inversion, realized by three separate layers of software

The output of the first software layer may be considered as deconvolved "primary" data that has been recorded at a non-reflecting, homogeneous data acquisition surface.

In software layer 2 the pre-processed data should first be used to determine the parameters of the macro subsurface model. In its simplest version the velocity $\bar{c}_{p}$ of each layer of an initial macro model is adapted such that the final estimate is fully consistent with the $\left(\mathrm{V}_{\mathrm{st}}, \mathrm{T}_{0}\right)$ values measured from the seismic data. In a more advanced version, the hyperbolic assumption is not used and a coherency analysis on CDP gathers is carried out. For the acoustic case, information on shear velocity $\bar{c}_{S}$ cannot be obtained from the data. Instead, use should be made of a heuristic relation for each macro layer such as

$\bar{c}_{p}=c_{0}+\alpha \bar{c}_{S}$

"Mudrock line".

Similarly, for the macro density $\bar{\rho}$, use can be made of

$\log \bar{\rho}=\log \rho_{0}+\beta \log \overline{c_{p}} \quad$ "Gardner relation"

for each macro layer.
The processing in software layer 2 is largely based on wave theory. The objective is to transform multi-offset primary data to angle- dependent reflectivity for each subsurface point at depth level $\mathrm{z}_{\mathrm{m}}$ and for each depth level of interest. This is done in the pre-stack migration process, which requires inversion for propagation operators $\mathbf{W}^{+}\left(\mathrm{z}_{\mathrm{m}}, \mathrm{z}_{0}\right)$ and $\mathrm{W}^{-}\left(\mathrm{z}_{0}, \mathrm{z}_{\mathrm{m}}\right)$. It can be shown (Berkhout, 1985) that inversion for downward propagation operator $\mathbf{W}^{+}\left(\mathrm{z}_{\mathrm{m}}, \mathrm{z}_{0}\right)$ involves a spatial deconvolution process on the common detector gathers; inversion for upward propagation operator $\mathbf{W}^{-}\left(\mathrm{z}_{0}, \mathrm{z}_{\mathrm{m}}\right)$ involves a spatial deconvolution process on the common source gathers. However, both spatial deconvolution processes can be combined into one processing scheme for each shot record! It is now generally accepted that pre-stack migration per shot record is the way to go. In pre-stack migration it is common practice to reduce the migration output to one stacked reflection coefficient for each subsurface grid point (depth point), the stack being carried out over all available angles of incidence at each depth point (CDP stack). Hence, in CMP processing common midpoint stacking is applied first, generally followed by zero-offset migration. In CDP processing multi-offset migration is applied first, generally followed by common depth point stacking. Moreover, in CDP processing the coherency analysis on CMP gathers (to determine stacking velocities) is replaced by a coherency analysis on CDP gathers (to verify and update the macro model).

The angle-dependent subsurface reflectivity defines the input for the third software layer. As a start (step 3a), the angle-dependent reflectivity information of the target zone is selected and inverted to velocity and density information $\left(c_{p}, c_{s}, \rho\right)$ for each subsurface point, using the expression for the angle-dependent reflection coefficient, trend information (from the macro model) and, if available, cross plots between $c_{p}, c_{s}$ and $\rho$ in the target zone. Note that cross plots may play a very important role in information based stabilization of inversion. Note also that a cross plot between $p$ and $c_{p}$ has the important advantage that it will formulate 
a Gardner type relation that includes covariance information (stochastic reformulation), allowing the solution to be off the mean curve.

Finally, in the last inversion step (step 3b) the velocity and density information in the target zone is used to estimate

- the rock parameters

$\begin{array}{lr}\rho_{S} & \text { density of the solid } \\ C_{S} & \text { compressibility of the solid } \\ \sigma(z) & \text { Poisson ratio of the bulk } \\ \beta(z) & \text { frame strength factor }\end{array}$

- pore volume parameters

$\phi$

$\mathrm{S}_{\mathrm{W}}$

water saturation

- pore fluid parameters

$\begin{array}{lr}\rho_{W} & \text { density of water } \\ C_{W} & \text { compressibility of water } \\ \rho_{h c} & \text { density of gas and/or oil } \\ C_{h c} & \text { compressibility of gas and/or oil. }\end{array}$

The inversion algorithm makes use of Gassmanntype equations for the compressional and shear velocity, the volumetric average equations for the bulk density and the fluid compressibility, a linear change in depth for the Poisson ratio, a semiempirical depth function for the frame strength factor (Geertsma, 1961) and any other empirical relation(s) available. First results show that lithological inversion is only feasible if a significant amount of additional (nonseismic) information is used!

Figure 8 summarizes the stepwise approach to seismic processing (acoustic version). It may be considered as a functional outline for the new generation of seismic "software. In the central second step propagation effects and reflection information are separated (by pre-stack migration). The validity of this critical and most time consuming part in seismic processing can be assessed by the evaluation of common depth point gathers. If, for a depth point in the target zone, the reflectivity information from different shot records are not aligned then inversion step 3 should not be started, but step 2 must be repeated with an updated macro model (user interaction).

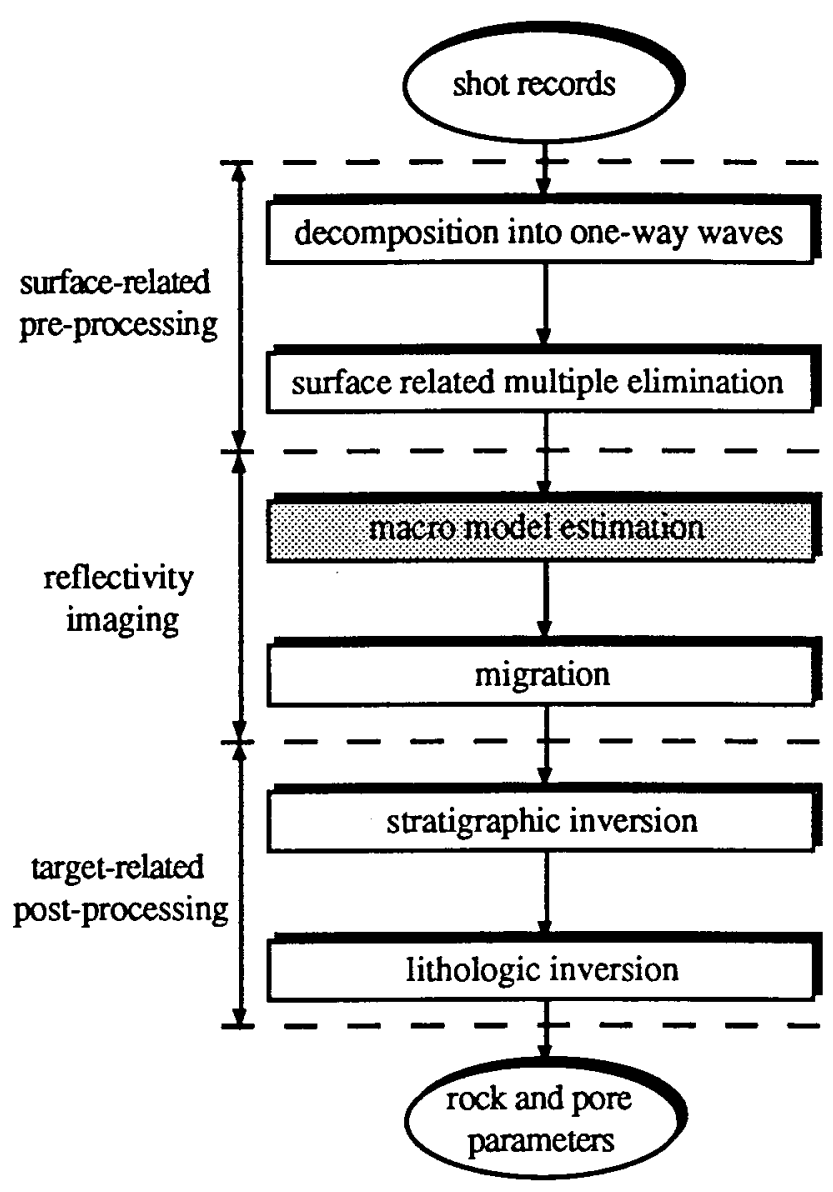

\section{Figure 8}

Acoustic version of the stepwise seismic inversion scheme; in this contribution the focus is on macro model estimation.

\section{Stepwise approach to elastic inversion}

The inversion process in step 3 is very difficult to carry out if only longitudinal reflectivity information (PP) is available. For an accurate estimation of the detailed shear velocity $c_{s}$, use of the shear reflection coefficient (SS) is essential. To a lesser extend, this also applies to the conversion reflection coefficients (SP and PS).Hence, step 3 makes it essential to follow the elastic approach. This means, on land, that ultimately three 
component sources and three component detectors must be used, and that, in tum, will result in nine different data sets and each will contain a mixture of $P$ - and $S$-wave data.

Figure 9 shows the elastic version of the stepwise seismic inversion scheme. Note that the $P$ - and $S$ - wave macro models are estimated independently after decomposition of the multi-component data into $P$ - and $S$-wave data.

In the following the acoustic version of the surfacerelated pre-processing and macro model estimation will be applied to the Marmousi data.

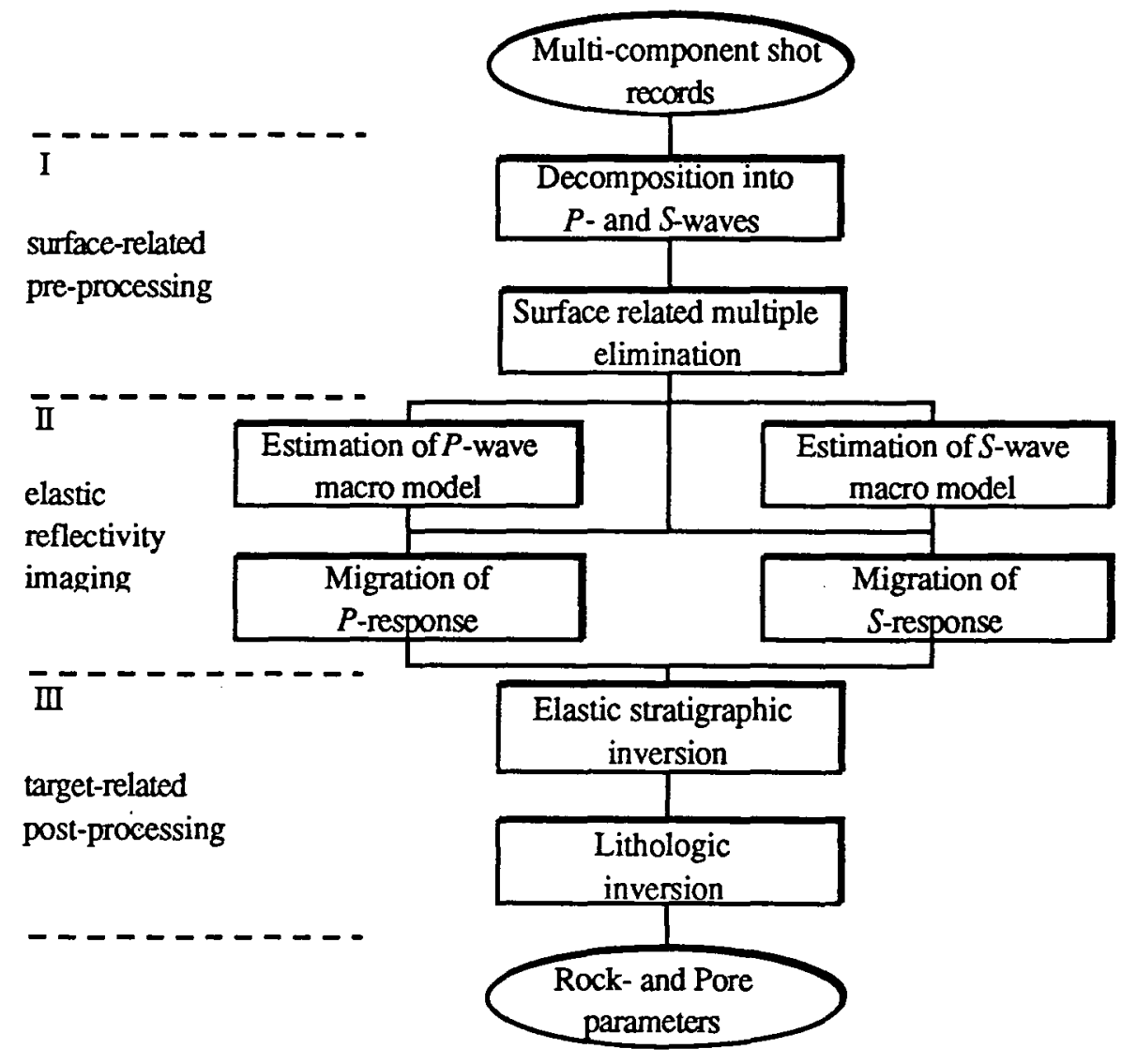

Figure 9

Elastic version of the stepwise seismic inversion scheme 


\section{MULTIPLE ELIMINATION}

\section{Introduction}

In contrast to the "conventional" dereverberation methods, which only remove reverberations that are generated in the surface layer, the DELPHI method removes the aforementioned reverberations as well as the multiples that are generated by the surface itself. This is accomplished by simulating a zero surface reflection coefficient. The main characteristic of this method is the fact that the seismic data itself is used as multiple predicting operator; knowledge about the subsurface is not required!

A publication on the surface related multiple elimination method can be found in Kennett (1979) for 1-D media and Berkhout (1985) for laterally varying media using the matrix formulation. Verschuur et al (1990) showed some examples of an adaptive version of the 2-D scheme, simultaneously estimating the source wavelet and removing the surface related multiples. Figure 10a schematically shows the effect of the surface related multiple elimination: removing the influence of the free surface, making the surface fully transparent (i.e. zero reflectivity).

In the Marmousi model there is a complex surface reflectivity, caused by a thin water layer of about $30 \mathrm{~m}$. To eliminate the full multiple generating effect of this complex surface, we extended the DELPHI multiple elimination method as follows. First the thin layer reverberations are removed at the source and receiver side with a layer dereverberation technique based on wave field extrapolation. Next, the surface related multiple elimination is applied, taking into account the complex reflectivity of the thin water layer for upward travelling waves. With this procedure the influence of the first layer is fully removed from the seismic data. Figure 10b schematically shows the effect of this extension: the removal of the influence of the first layer.

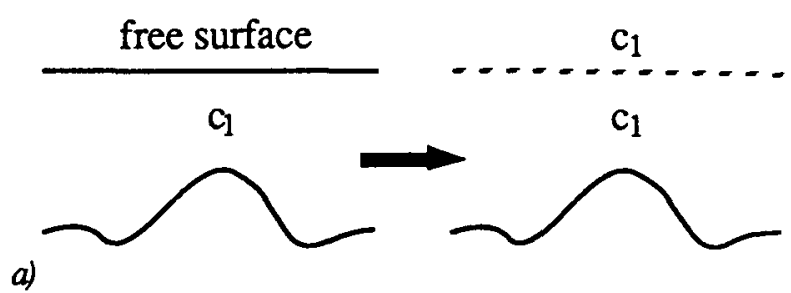

b)

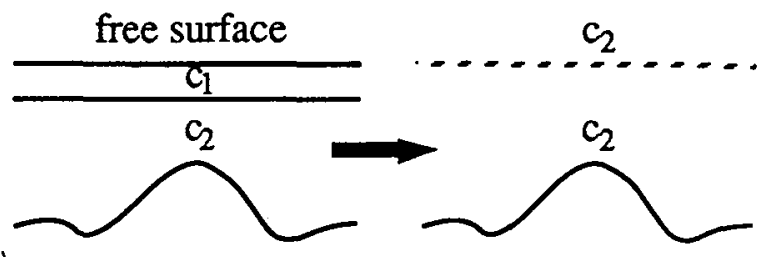

Figure 10

a) Effect of surface related multiple elimination: removal of the influence of the free surface. $b$ ) Effect of the extended multiple elimination method: removal of the influence of a thin water layer.

\section{Multiple elimination method}

In this section the theory for the surface related multiple elimination in the presence of a thin surface layer (the $30 \mathrm{~m}$. water layer in the Marmousi model) is reviewed. For a more mathematical description of the method we refer to Verschuur et al. (1990). First we introduce a model to describe the response of a subsurface with a thin surface layer. Figure 11 describes this model.

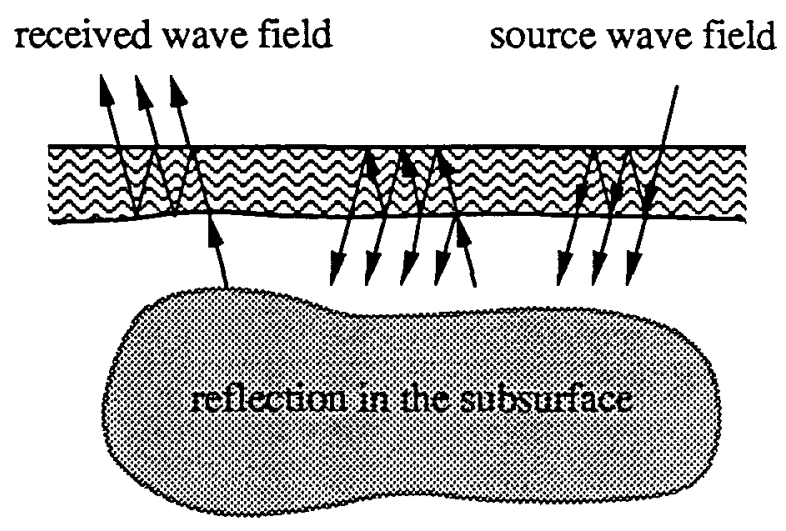

Figure 11

Seismic response in the presence of a thin near surface layer. 
At the right hand side of Figure 11 we see the downgoing illuminating source wave field. This wave field will be trapped in the thin layer before it propagates into the subsurface, which means adding some thin layer reverberations at the source side. After that the wave field is reflected in the subsurface and the reflected wave field propagates upward to the surface again, which is visible in the middle and at the left hand side of Figure 11. When it arrives again in the thin surface layer, reverberations will be added; the data will be detected (left hand side) and reflected into the subsurface again (middle), generating new multiples etc.

To fully remove the influence of the thin surface layer on the data we propose a two-step procedure. In the first step we remove the thin layer reverberations at the source and the receiver side. This can be done by a wave field prediction and subtraction technique similar to the approach of Berryhill and Kim (1986). By extrapolating the received data through the first thin layer once, primaries become first order reverberations, first order will become second order reverberations etc. Subtracting this from the original data will yield the data without the thin layer reverberations. This procedure should be applied once on common shot gathers (receiver side reverberations) and once on common receiver gathers (source side reverberations).

received wave field source wave field

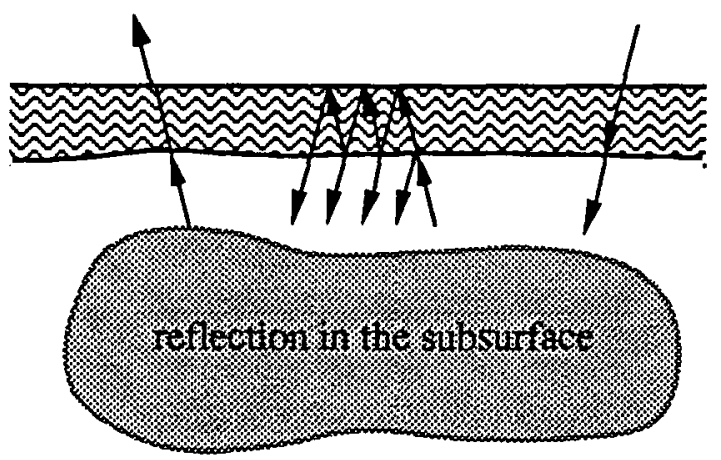

Figure 12

Seismic response after applying the thin layer dereverberation technique.
Anyway, whatever method is used, after dereverberation the response from Figure 11 will be transformed into a response as visualised in Figure 12. After this dereverberation the second step is applied, which is a surface related multiple elimination as described in the introduction. For the situation with a thin surface layer the method has to be adapted, because remaining multiples are related to the free surface in combination with the thin layer bottom (Figure 12, middle). To remove these multiples, the reflectivity to be used in the multiple elimination scheme should not be the free surface reflectivity $(\mathrm{r}=-1)$ but the angle dependent reflectivity of the thin layer from below, including the thin layer reverberations. Taking this into account all surface related multiples can be eliminated and the remaining response is as illustrated in Figure 13. Note that after these two steps both the free surface as the thin layer bottom can be considered as transparent.

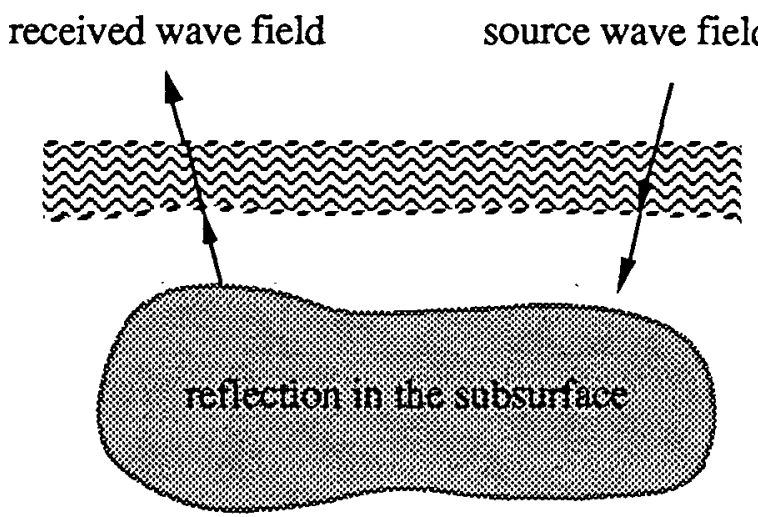

Figure 13

Seismic response after applying the surface related multiple elimination method, including the angle dependent reflectivity of the thin layer.

\section{Multiple elimination applied on the Marmousi dataset}

The two-step procedure of removing the water layer reverberations and after that the removing of the surface related multiples has been applied on the Marmousi dataset. Figure 14a shows shot 40 of the Marmousi dataset. It is clear that all primaries suffer from the reverberations of the water layer, because the wavelet shows a ringing character. After 
applying the dereverberation method the result for shot 40 is shown in Figure 14b. The wavelet of the primaries is much more clear, especially in the lower part of the shot record. Figure 15a and 15b show the dereverberation result for shot 200. Again the result of the dereverberation is clear. Also some $\mathrm{f}-\mathrm{k}$ filtering has been applied, which is visible for the primaries in the top of the shot records. After the dereverberation the surface-related multiple elimination method was applied to remove the multiples related to the combination of free surface and sea bottom. The result for shot 200 is shown in Figure 15c. There does not seem to be much difference compared to Figure $15 \mathrm{~b}$. Some weak events indicated with arrows in Figure 15b, which are surface related multiples, have been removed indeed. Apparently, the amplitudes of the surface-related multiples in the Marmousi model are small compared to the amplitudes of the primaries within the registration time. This means that the contrasts below the water bottom are not very large. Note that the small improvement in applying the surface related multiple elimination is not a deficiency of the method but has to do with the model. Therefore we did not apply this multiple elimination to all other shots. Unfortunately the multiples were too weak to get a full estimate of the source wavelet, but at least it was possible to estimate a general time shift of the source wavelet which appeared to be $52 \mathrm{~ms}$. We applied this time shift to the data, to end up with a wavelet as close as possible to a zero-phase wavelet. To see an overall result of the dereverberation a commonoffset section for the nearest offset of $200 \mathrm{~m}$ is shown in Figure 16a before and in Figure 16b after dereverberation.

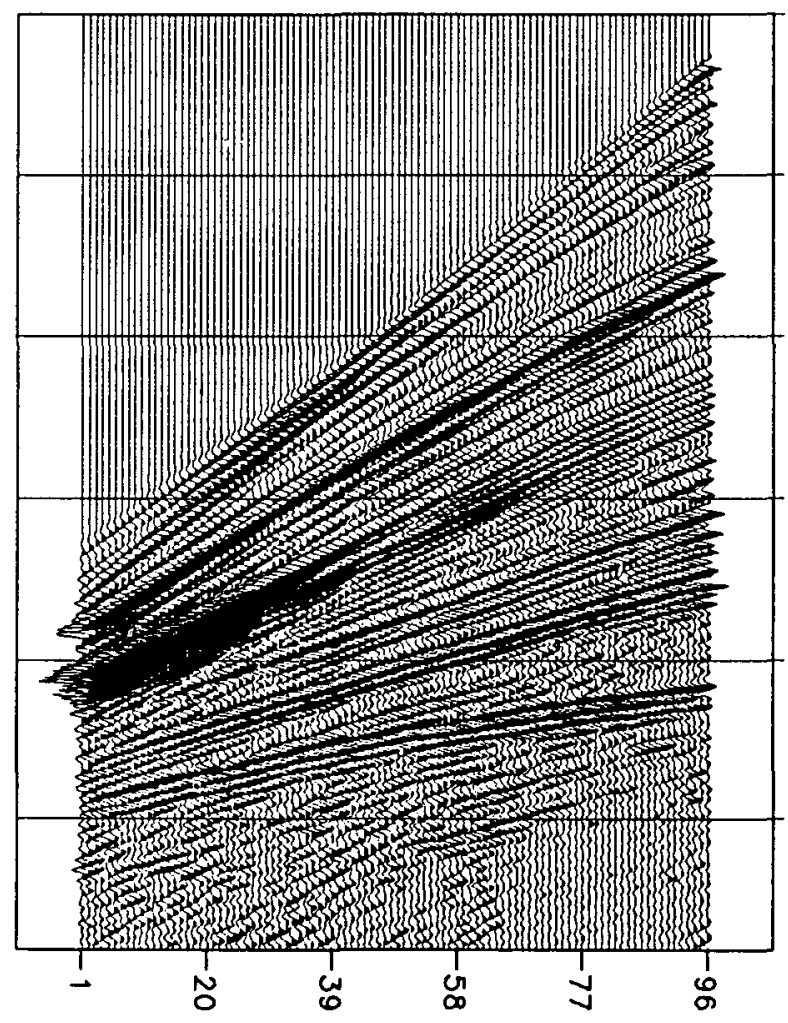

a)

Figure 14

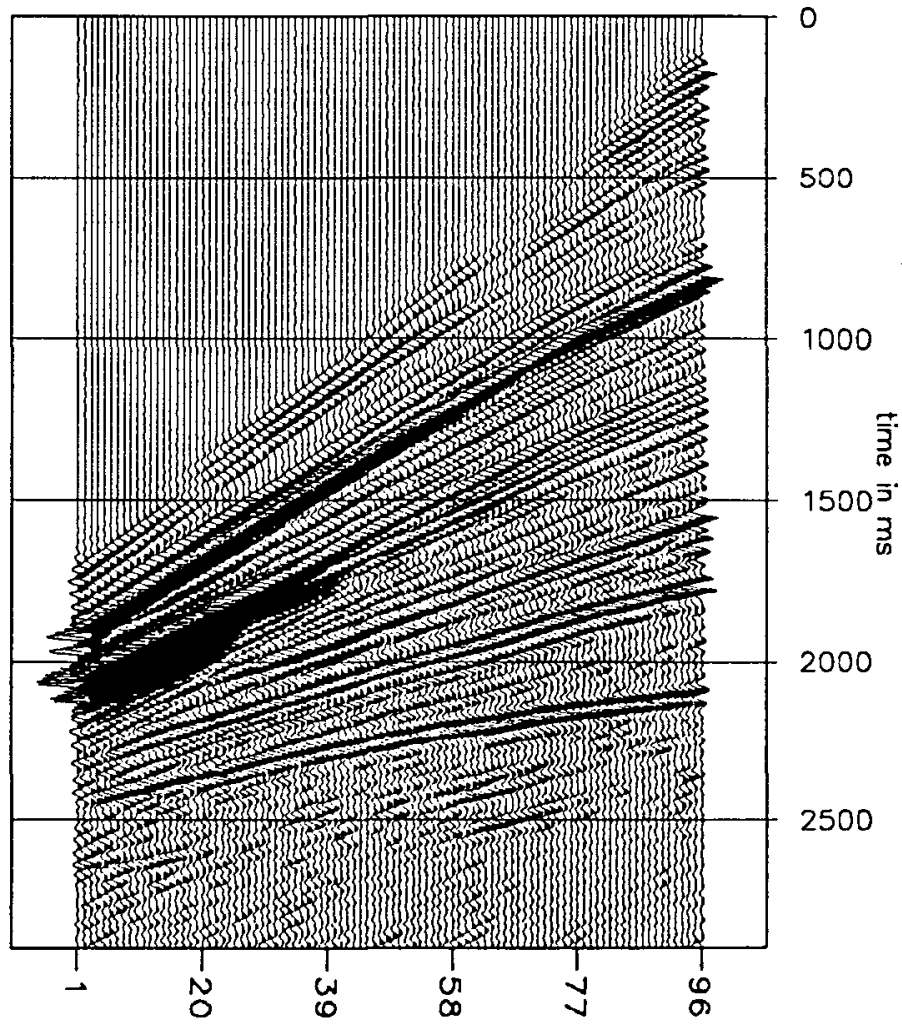

b)

a) Shot 40 of the Marmousi dataset. b) Shot 40 after applying the dereverberation method. 

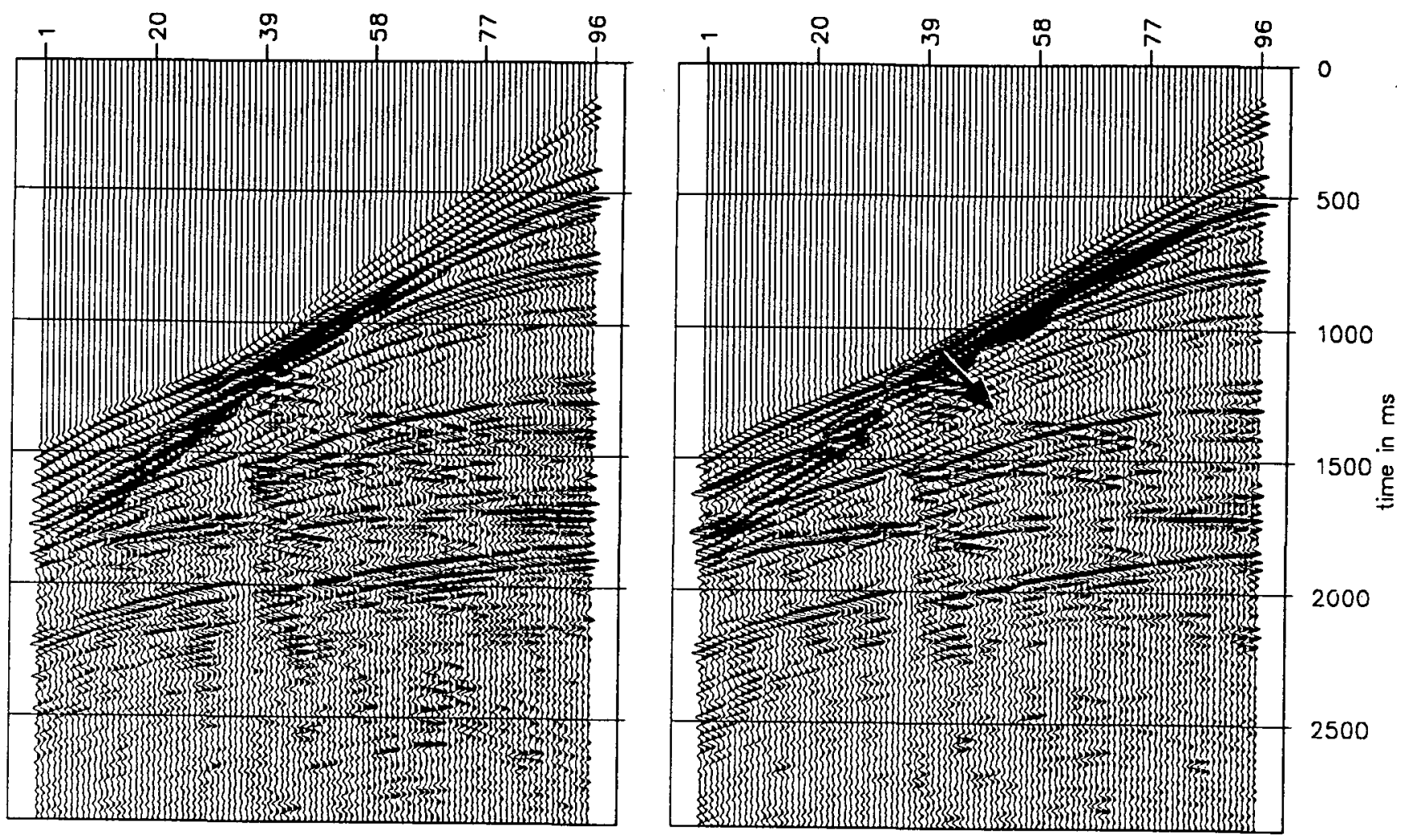

a)

b)

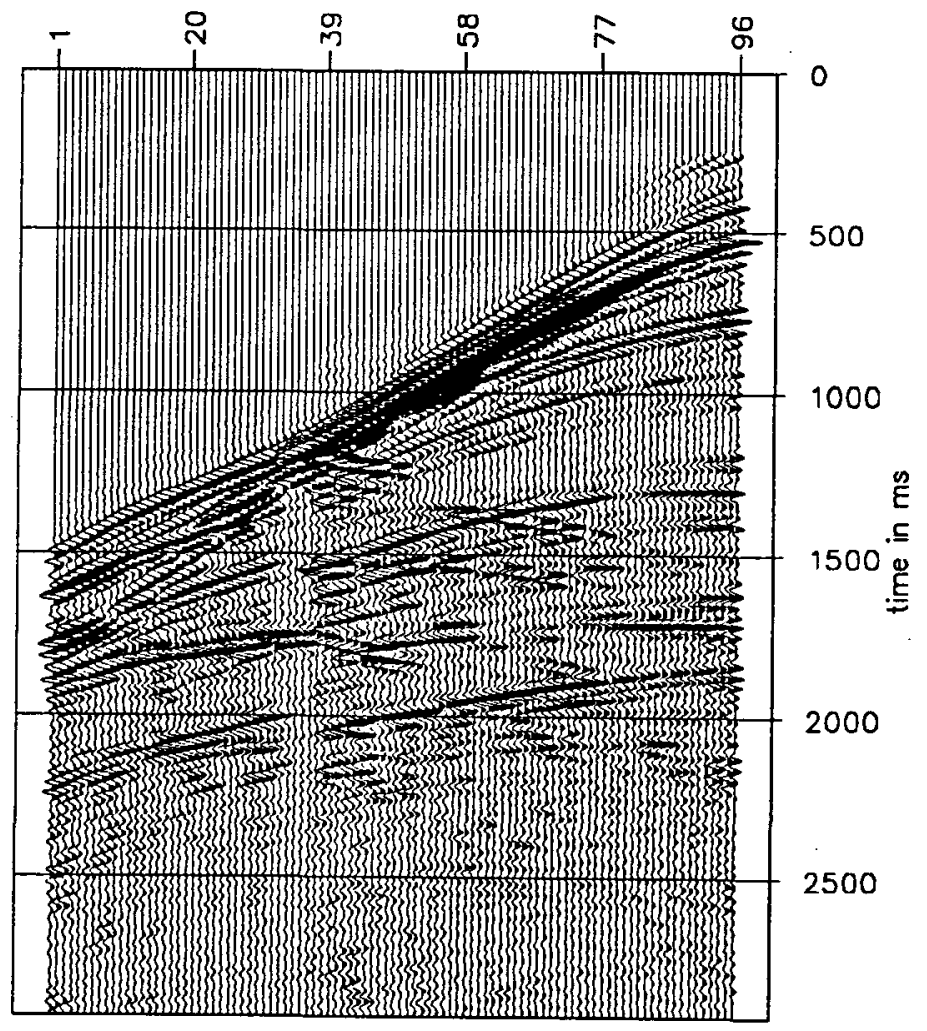

\section{Figure 15}

a) Shot 200 of the Marmousi dataset. b) Shot 200 after applying the dereverberation method. c) Shot 200 after applying the dereverberation method and the interface related multiple elimination. Note that the data contains very little multiple energy. The arrow in $b$ ) indicates a surface related multiple. 

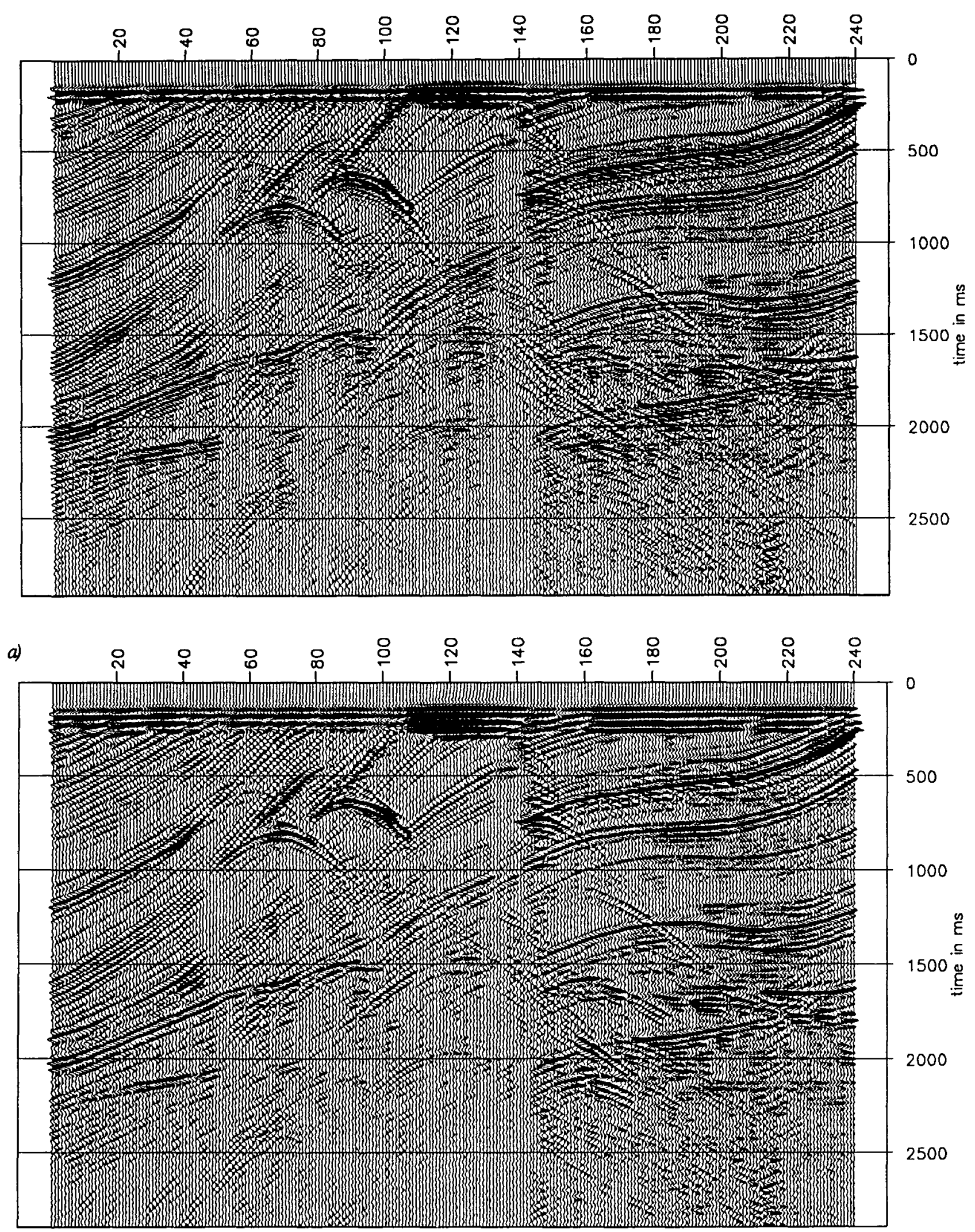

b)

Figure 16

a) Common-offset section (200 m) of the Marmousi dataset. b) Common-offset section (200 m) after applying the dereverberation method. 


\section{ESTIMATION OF MACRO SUBSURFACE MODELS}

\section{Introduction}

In the DELPHI scheme, the data after surfacerelated preprocessing are used to separately estimate the $\mathrm{P}$-wave macro model and, if applicable, the Swave macro model. We start with an overview of the macro model estimation method. Next, the application to the Marmousi data ( $\mathrm{P}$-waves only) is discussed.

\section{Overview of the macro model estimation method}

The Philosophy.

Prestack depth migration algorithms are very sensitive to the accuracy of the macro model. Therefore, the philosophy is to use this sensitivity for the estimation of macro models. This concept is nowadays well appreciated and different algorithms have been developed which employ the underlying idea. Jeannot et al. (1986) use S-G depth extrapolation and analyze focussing of the extrapolated data before imaging. Since stacking over the shots is done implicitly in this scheme (unlike in shot record migration) it is not possible to analyze the data before summation over the shots. The DELPHI method (Cox, 1991) is based on the coherency analysis of Common Depth Point gathers, as obtained by shot record redatuming. This means that in the DELPHI method the data is analyzed before imaging and before summation over all shots!

\section{The Method.}

In the above mentioned technique of Jeannot, extrapolation is done in a recursive way and consequently all depth points in the subsurface are involved. This allows a laterally continuous velocity estimation, but it is quite expensive in 2-D and practically unfeasible in 3-D. In our opinion it is not necessary to do a laterally continuous velocity analysis since within one macro layer the velocity does not change rapidly. The macro boundaries (e.g. faults), however, can have sudden changes. Therefore our approach is to split the estimation of the macro model in two parts:

Estimation of the macro layer velocities. This is the most expensive part of the procedure. It involves extrapolation of shot records to 'vertical datum lines' (as will be explained in detail below).

Estimation of the macro boundaries. Once the macro layer velocities are known, the macro boundaries can be estimated straightforwardly from the traveltimes. Since in this stage we are not interested in obtaining true amplitudes but merely in delineating the structure of the macro boundaries, a fast time-to-depth conversion algorithm can be chosen.

The two estimation modules (velocities, boundaries) together define our macro model estimation procedure. Depending on the objective they can be used in a bore hole-oriented estimation scheme or in a reservoir-oriented scheme. In the bore hole-oriented scheme the velocity analysis is done for one lateral position (vertical datum line) at the location of a potential well (Figure 17a). In the reservoir-oriented scheme the velocity analysis is done for a number of lateral positions because of the lateral extent of the problem (Figure 17b). In general, the velocity analysis can be sparsely sampled in the lateral direction of one macro layer (just as with conventional velocity analysis). 
a)
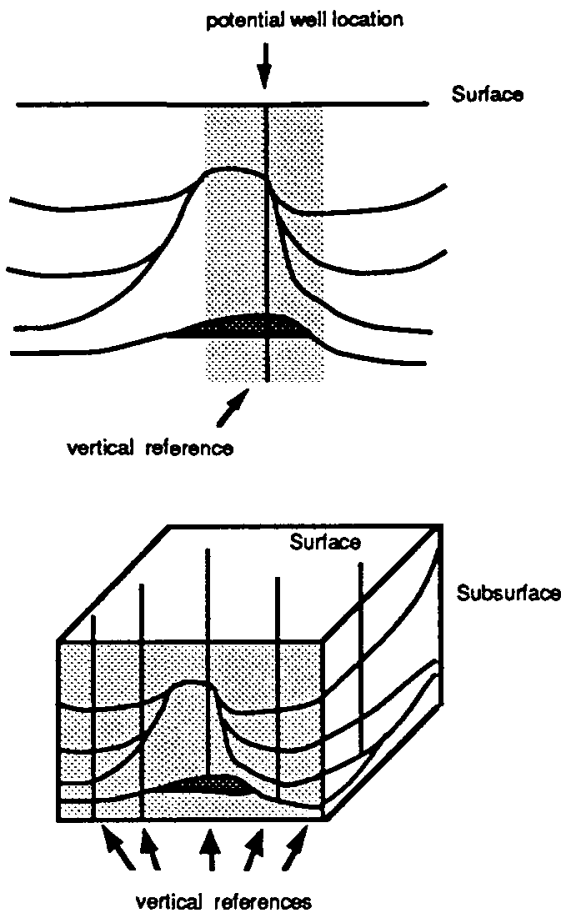

b)

\section{Figure 17}

a. A bore hole-oriented estimation scheme involves velocity analysis at a potential well location ('vertical datum line').

b. In a reservoir-oriented estimation scheme velocity analysis is done for a number of vertical datum lines.

The total procedure is most simply implemented in a layer-stripping mode (Figure 18a). This is however not the most economical implementation as was also recognized by MacBain (1989). The total number of iterations can be reduced by applying a cascaded velocity estimation (Figure $18 b)$. Here per iteration the interval velocities for a number of macro layers are estimated. Although the estimation for a deep layer is less accurate when shallower layers still contain errors, the overall number of iterations is reduced. Note that in both schemes the macro velocity estimation is followed by macro boundary estimation. In the following we will discuss these two estimation modules in more detail.

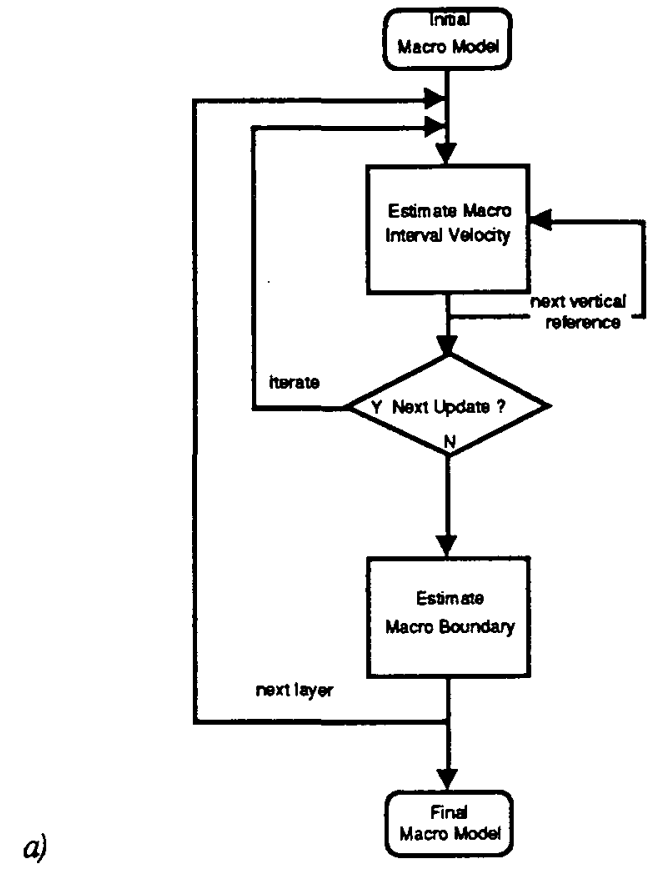

b)

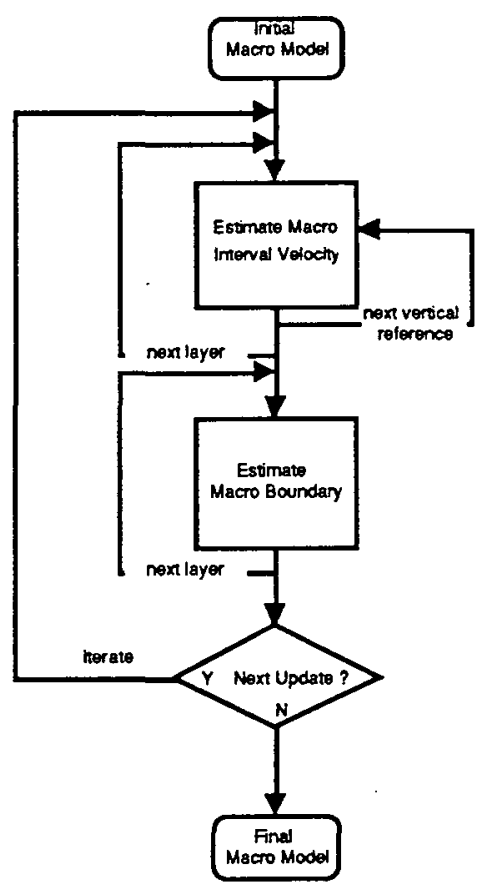

Figure 18

a. Reservoir-oriented estimation scheme in a layerstripping implementation.

$b$ Reservoir-oriented estimation scheme in a multi-layer (cascaded) implementation. 
Since the velocity within one macro layer does not change rapidly the velocity estimation may be sparsely sampled in the lateral direction. The estimation of the macro velocities involves extrapolation of the prestack surface data to predefined vertical datum lines. At each vertical datum line a velocity analysis is performed. The extrapolation is done per shot record (shot record redatuming). This enables us to analyze the redatumed data (unlike S-G schemes) before stacking over all shots. So with a shot recordoriented scheme genuine CDP gathers can be analyzed.

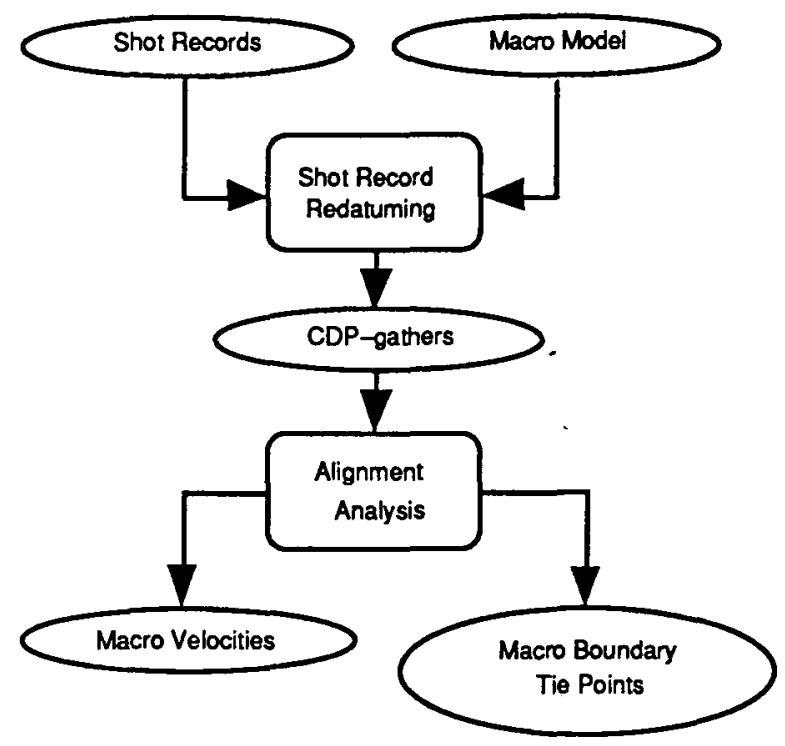

Figure 19

Estimation of the macro velocities involves shot record redatuming, followed by alignment analysis of genuine Common Depth Point (CDP-)gathers.

Redatuming of one shot record to one depth point in the subsurface results in one CDP trace (Figure 20). If the depth point is on a reflector and if the macro model used in redatuming is correct, then an event will occur at $t=0$. By gathering the traces of all redatumed shots at this particular depth point a genuine CDP gather is obtained (Figure 21a). If the macro model is correct and if the depth point is on a reflector then horizontal alignment of the events will occur at $t=0$. Note that

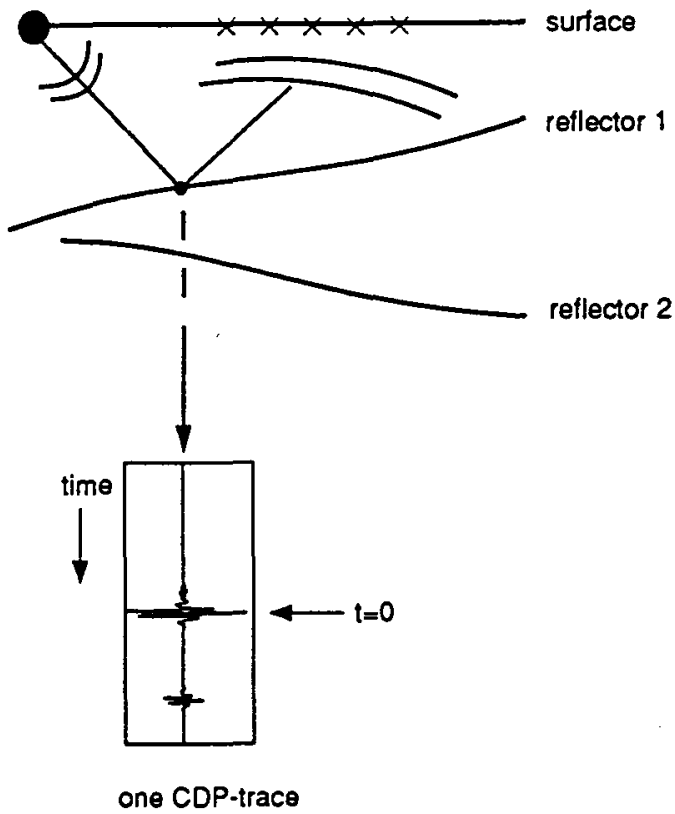

Figure 20

Redatuming of one shot record to one depth point in the subsurface yields one CDP trace.

the alignment is irrespective of the dip and curvature of the interface and irrespective of the complexity of the overburden! This alignment property is used in the updating procedure. One way of measuring the alignment is by a simple summation of all traces in a CDP gather (Figure 21b). This yields a true zero-offset trace registered at the particular depth point (Kinneging at al., 1989). If the depth point is located at a macro boundary and if the macro model is correct, the amplitude in the zero-offset trace will be maximum due to the alignment in the CDP gather. This is in accordance with the physical interpretation of the ZO trace: zero-offset acquisition on top of an interface yields the highest amplitude since no geometrical spreading is involved. Note also that the two way traveltime of such a $\mathrm{ZO}$ trace equals zero! 


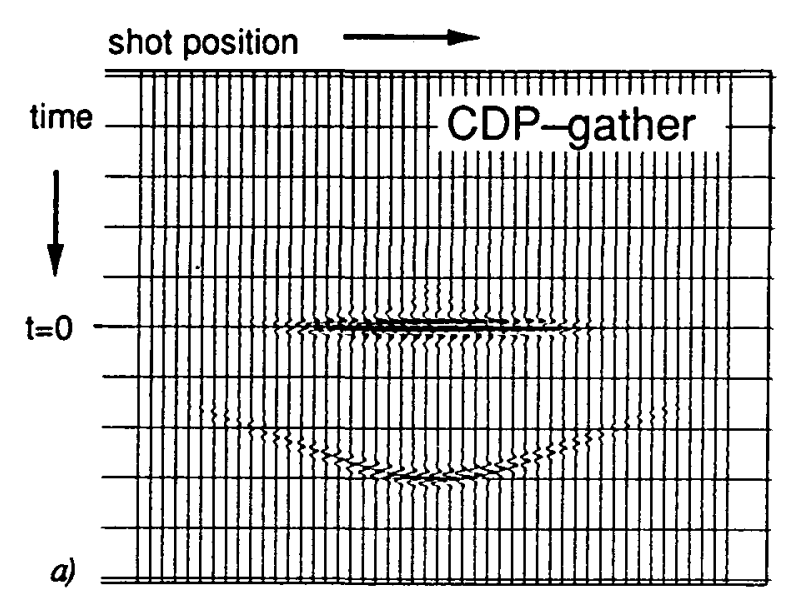

b)

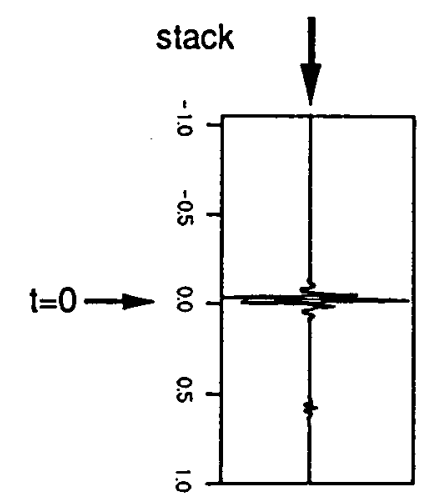

\section{Figure 21}

a. A CDP gather consists of the redatumed traces at one depth point due to many shots.

b. Stacking the traces in a CDP gather yields a true zero-offset trace at the considered depth point.

In conclusion, if the underlying macro model is correct, focussing of energy will occur in the $\mathrm{ZO}$ traces at interface depth points and at zero time due to alignment of the events in the corresponding CDP gathers. If the macro model is incorrect, focussing will still take place but not at an interface depth point and not at zero time. By redatuming to a range of depth points on a vertical datum line a panel of ZO traces can be obtained (after CDP stacking) which is a function of the two-way zerooffset time and the depth (of the depth point) as shown in Figure 22a. By contouring the envelopes of these data the focussing of energy can be elegantly shown (Figure 22b). By picking the focus location the velocities can be updated (Jeannot et. al., 1986; Cox , 1991).

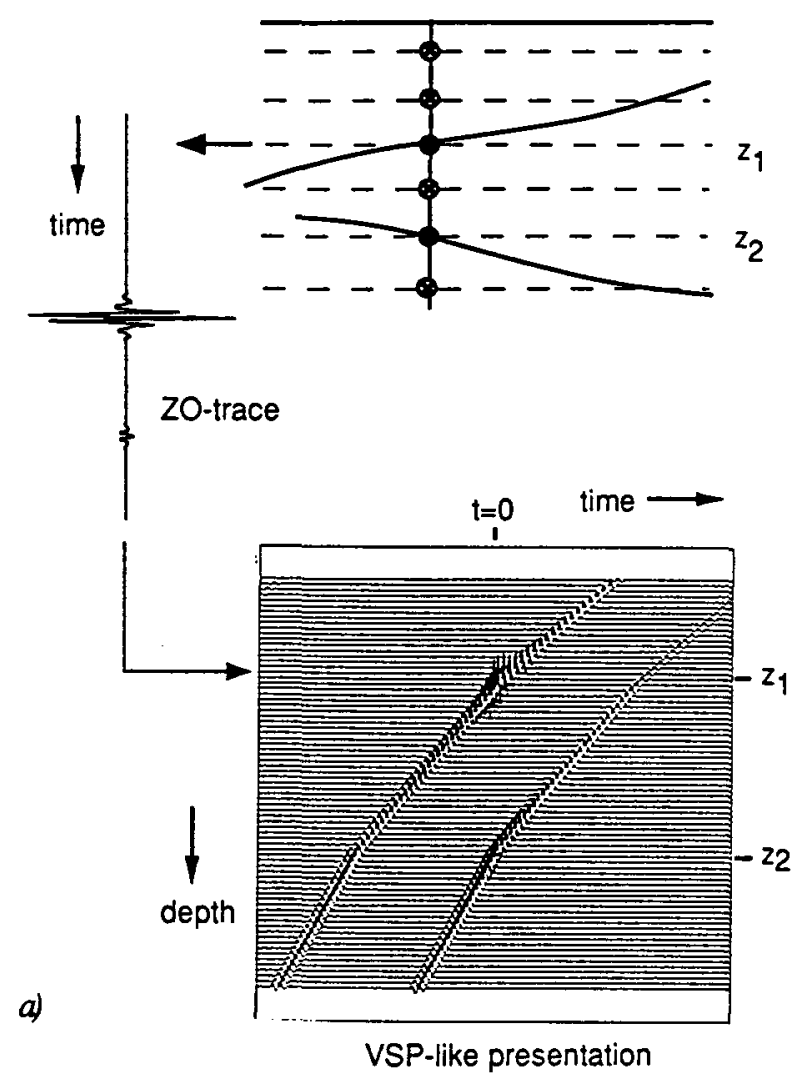

b)

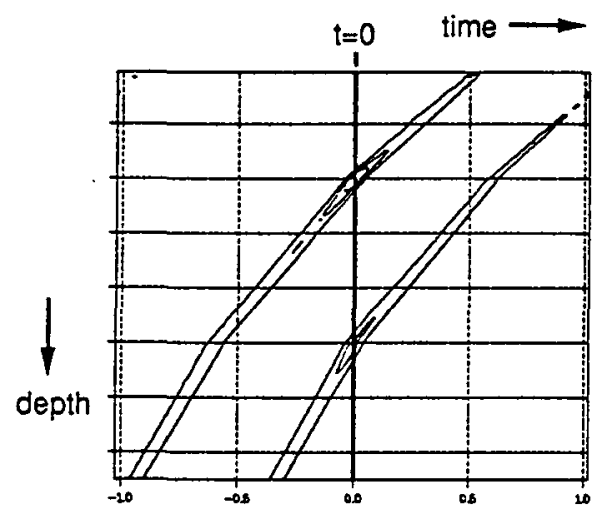

Figure 22

a. Redatuming to the depth points on a vertical datum line, followed by CDP stacking, yields a panel of zerooffset traces that can be displayed in a VSP-like presentation as a function of two way travel time (horizontal axis) and depth (vertical axis).

$b$. Contouring the (envelope of the) data of figure a elegantly visualizes the focussing of energy that occurs due to alignment of events in the corresponding CDP gathers. 


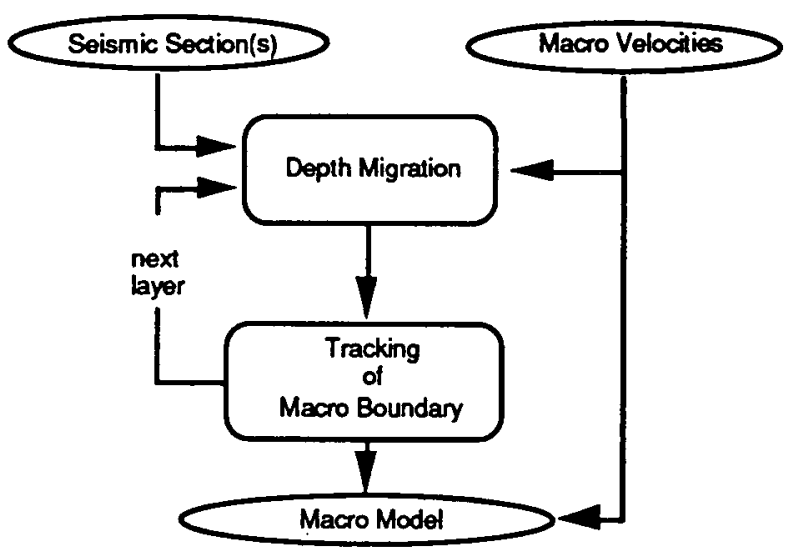

Figure 23

Estimation of the macro boundaries may be carried out by depth migrating the data to just below the macro boundary and then tracking the imaged macro boundary

\section{Estimation of the macro boundaries}

The strategy of obtaining the macro boundaries involves depth migration (Figure 23). Input to the depth migration algorithm are either the stacked data or a substack or merely a moveout-corrected singleoffset section. These data are then depth migrated, starting with the estimated velocity of the first macro layer, to just below the base of the first macro boundary (until the first macro boundary is completely imaged). This boundary is then tracked and put in the macro model file. Then the procedure is continued, but now the velocity contrast is taken into account and the migration is done to just below the next macro boundary which is then tracked, etc. Rapid changes of the macro boundaries are correctly taken into account.

\section{Macro model estimation on the Marmousi data}

The procedure described above was used to estimate the macro velocity model of the Marmousi data. The initial model was obtained by conventional velocity analysis on CMP-gathers (Figure 24). No effort has yet been spent in incorporating geologic information about the area. Zero-offset migration of the stacked section with the initial model shows that at the left and right side already a consistent image is obtained (Figure 25). For the center part of the migrated stacked section it is clear that in this region the initial macro model needs major repairs. This is confirmed by the focus panels shown in Figure 26; well-pronounced foci are visible near the $t=0$ axis for the panels near the sides of the model (Figs 26a and 26c), but the focus panel at the center (Fig. 26b) shows that the initial model is far from correct.

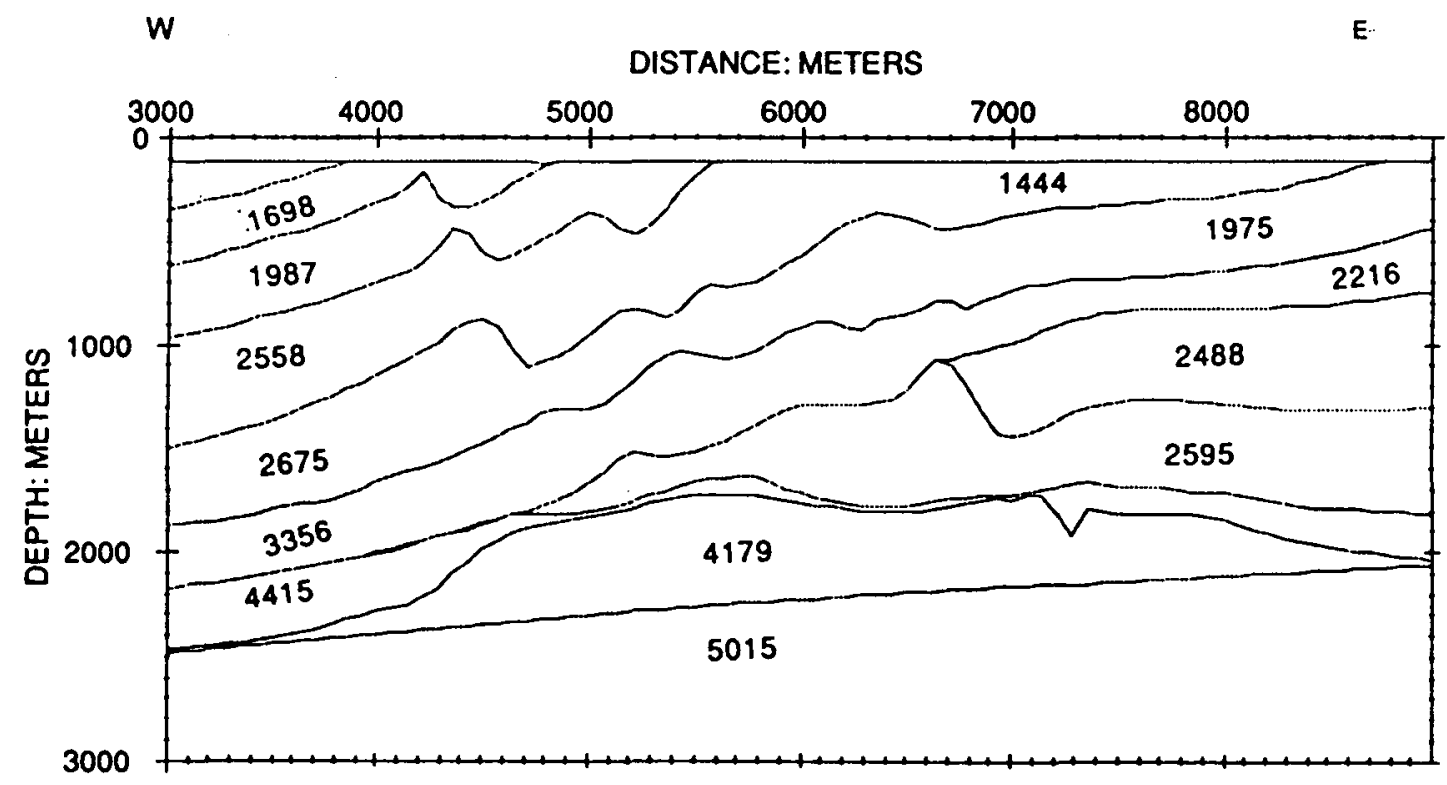

Figure 24

Initial model obtained by conventional velocity analysis on CMP gathers. 


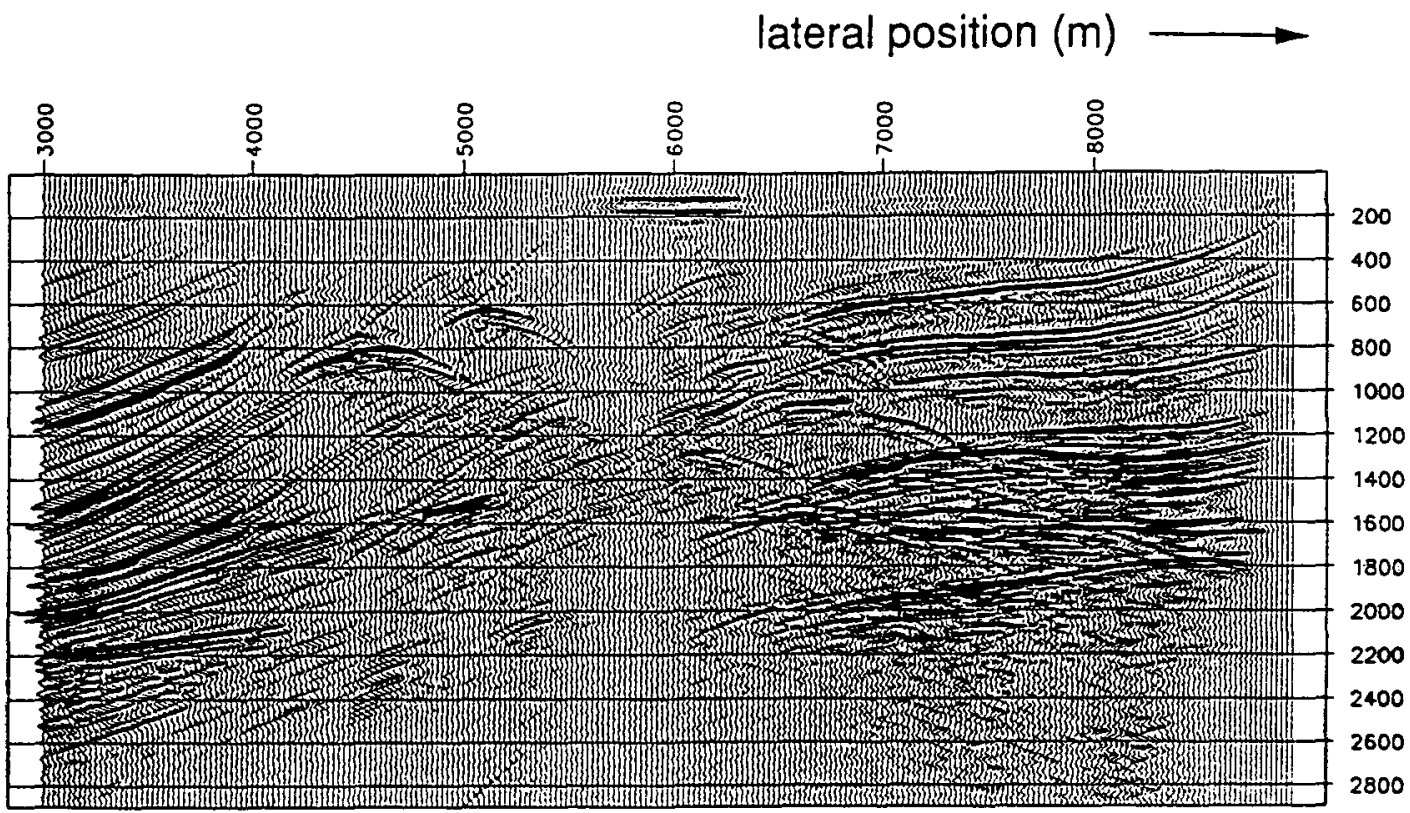

章

a)

lateral position (m)

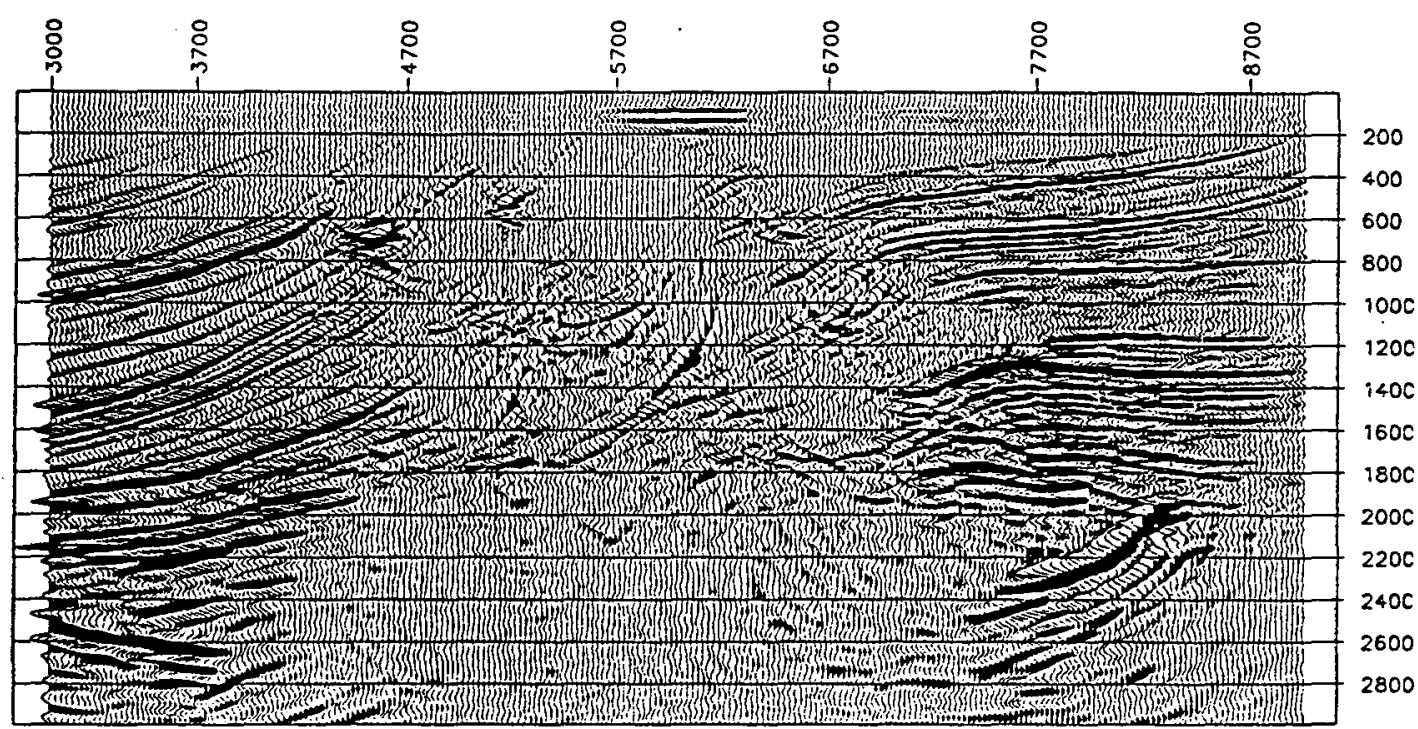

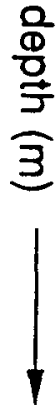

b)

Figure 25

a. Stacked section (with deconvolution and DMO).

b. Poststack depth migration of the stacked section. 


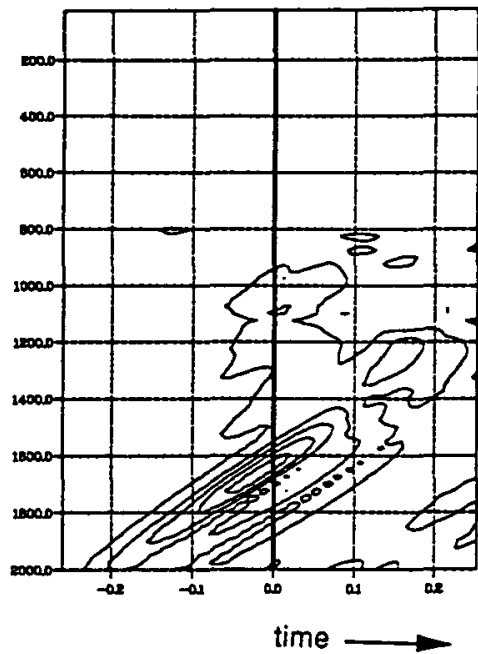

a)

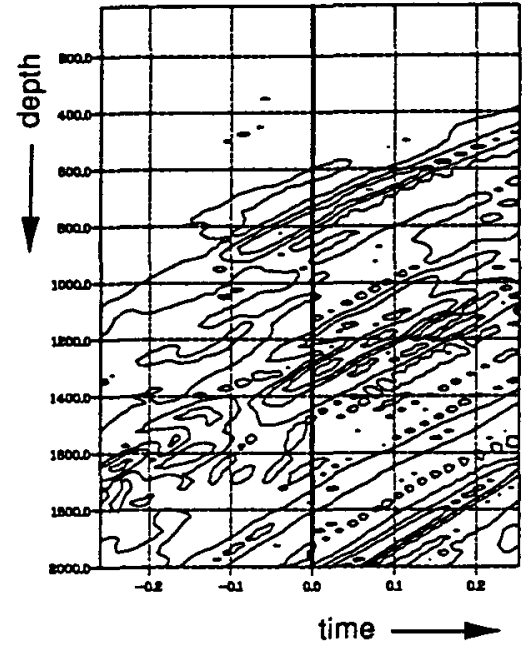

b)

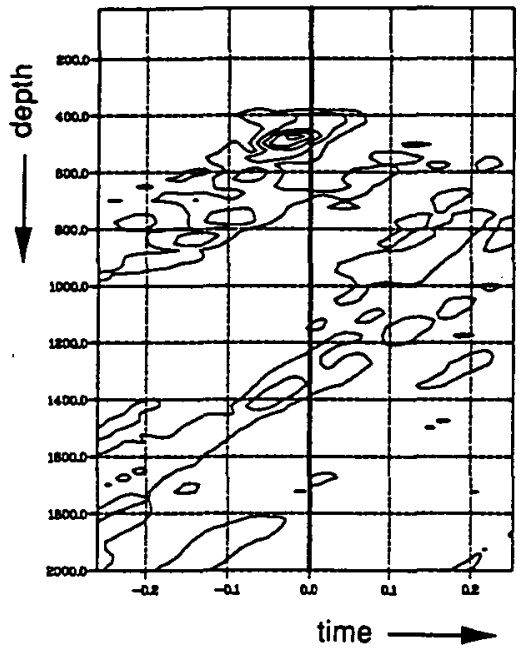

c)

Figure 26

Focus panels at $x=3000 \mathrm{~m}$. (a), $x=4000 \mathrm{~m}$. (b) and $x=7000 \mathrm{~m}$. (c), obtained by shot record extrapolation with the model of Figure 24. Note that only strong reflecting boundaries yield high amplitudes in the focus panels.

Hence, conventional velocity analysis breaks down in this complex subsurface structure. To tackle the complex problem encountered in the center region the boundaries on the left and on the right are estimated separately. By doing this we approach the problem area from two independent directions. The foci are picked from the focus panels and used to obtain updated macro velocities at both sides. Next, the macro boundaries need to be determined. This is done by depth migration of the zero-offset data shown in Figure 27. These data are obtained as a by-product of the multiple elimination procedure. Note that we did not use the stacked data since in complex subsurfaces these do not represent true zero-offset traveltimes. From these results the macro model shown in Figure 28a is obtained. As can be seen, this model is much simpler than the initial model. It contains only the strong reflecting boundaries (macro boundaries) that yield high amplitudes in the focus panels. At this stage we tried to incorporate the available geologic information that was provided to us. Unfortunately the original model description contained a disturbing error that seriously biased our interpretation (we were informed of this error only a few days before the date of the workshop). In the original documentation it was given that the deltaic sediment was "...thickening from east to west ...". From this we concluded that the sea was on the west side (left), since the sediment interval was supposed to be thicker on this side of the model. Consequently, land was assumed to be on the east side (right). Therefore, growth faults should be defined from bottom-left to top-right (all the way to the surface). Hence, the fault structures visible in the prestack migration (Figure 28b) were interpreted as small counter-regional faults, that terminate against a large growth fault. The updated model after focussing analysis combined with the erroneous interpretation was as displayed in Figure 29. However, in reality land is on the west side and sea is on the east side (the deltaic sediment was "...thickening from west to east ..."). Therefore, the faults that were interpreted as small counterregional faults should have been interpreted as large growth faults instead. After we received the correct geologic description, one update could be realized within the remaining time. 


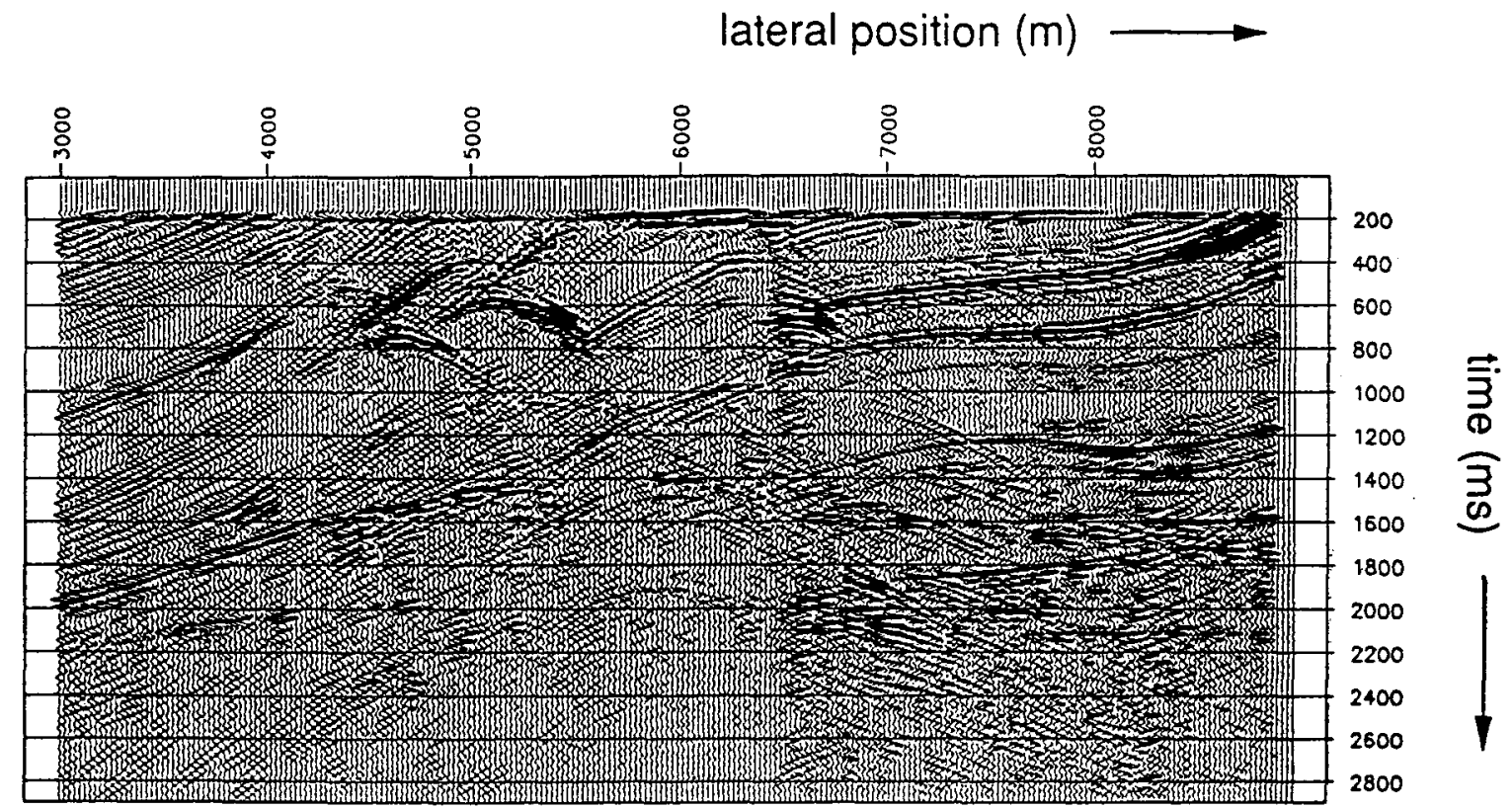

Figure 27

Zero-offset data after near-offset interpolation (obtained as a by-product of the multiple elimination).

The result is shown in Figure 30a (see also Colour figure 10).

The prestack migration with the model of Figure $30 \mathrm{a}$ is illustrated in Figure 30b. At the sides of the model we are confident in the result, since the focus panels calculated with this model contain foci near $t=0$. (Figure $31 \mathrm{a}$ and $31 \mathrm{c}$ ). Of course, in the center part more iterations are needed as illustrated by the focus panel in Figure 31b.

\section{CONCLUSIONS}

1. Horizontal alignment of macro reflections in CDP-gathers is an excellent criterion for verification and updating of macro models. This is confirmed by the fact that focus panels of the Marmousi data with the correct macro model represent well interpretable pictures (see the contribution by Blacquière).
2. After each update the data should be depth migrated with the new macro velocities to evaluate the macro boundaries. In this step we prefer to migrate the zero-offset data that are obtained in our surface-related multiple elimination step.

3. In complicated situations focus panels look like 'scattergrams' and it is impossible to converge to the correct solution with focus panels only. The process should be guided by macro geologic models of the area. Of course, the provided macro geologic information should be consistent with reality.

4. In complicated situations we also advise to use a layer stripping strategy. 


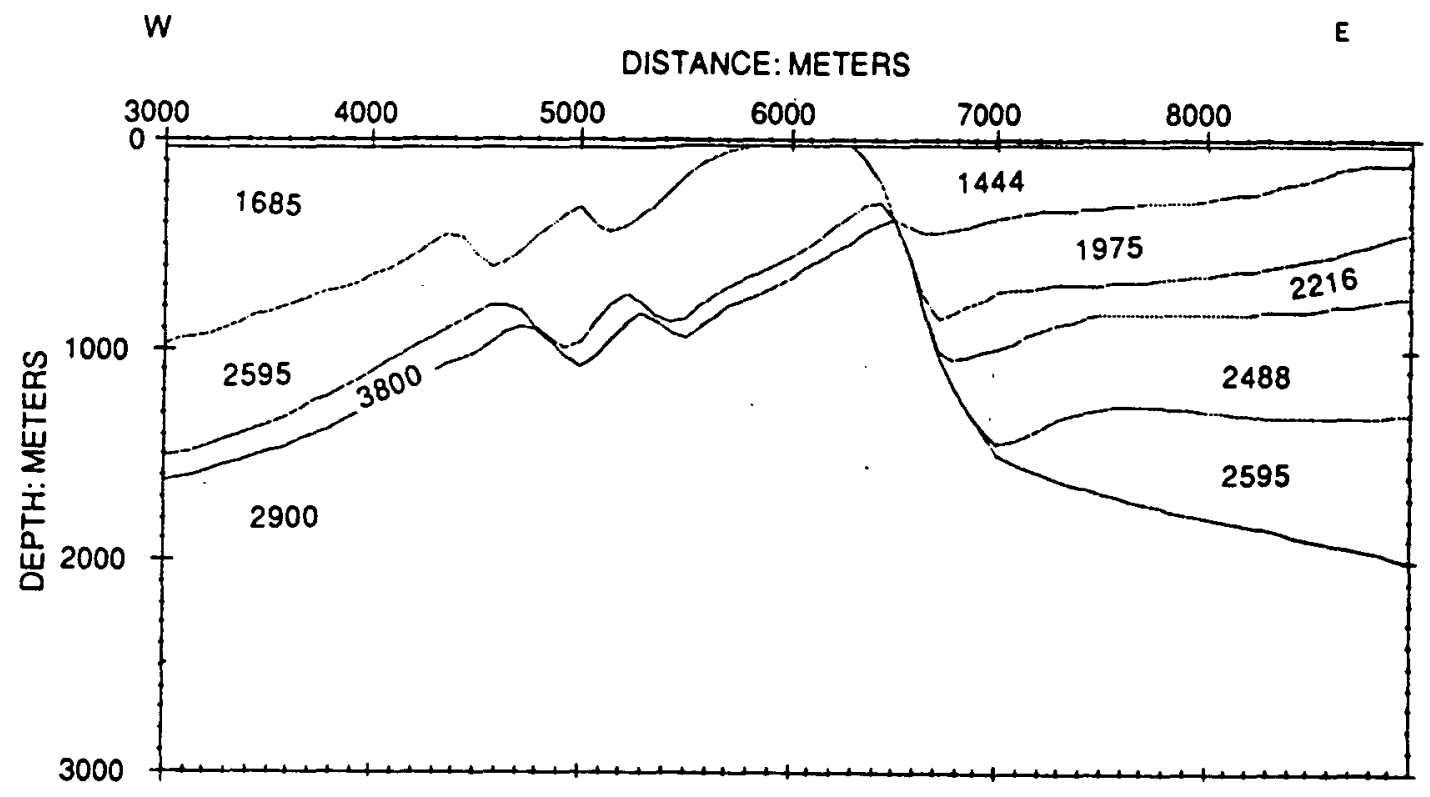

a)

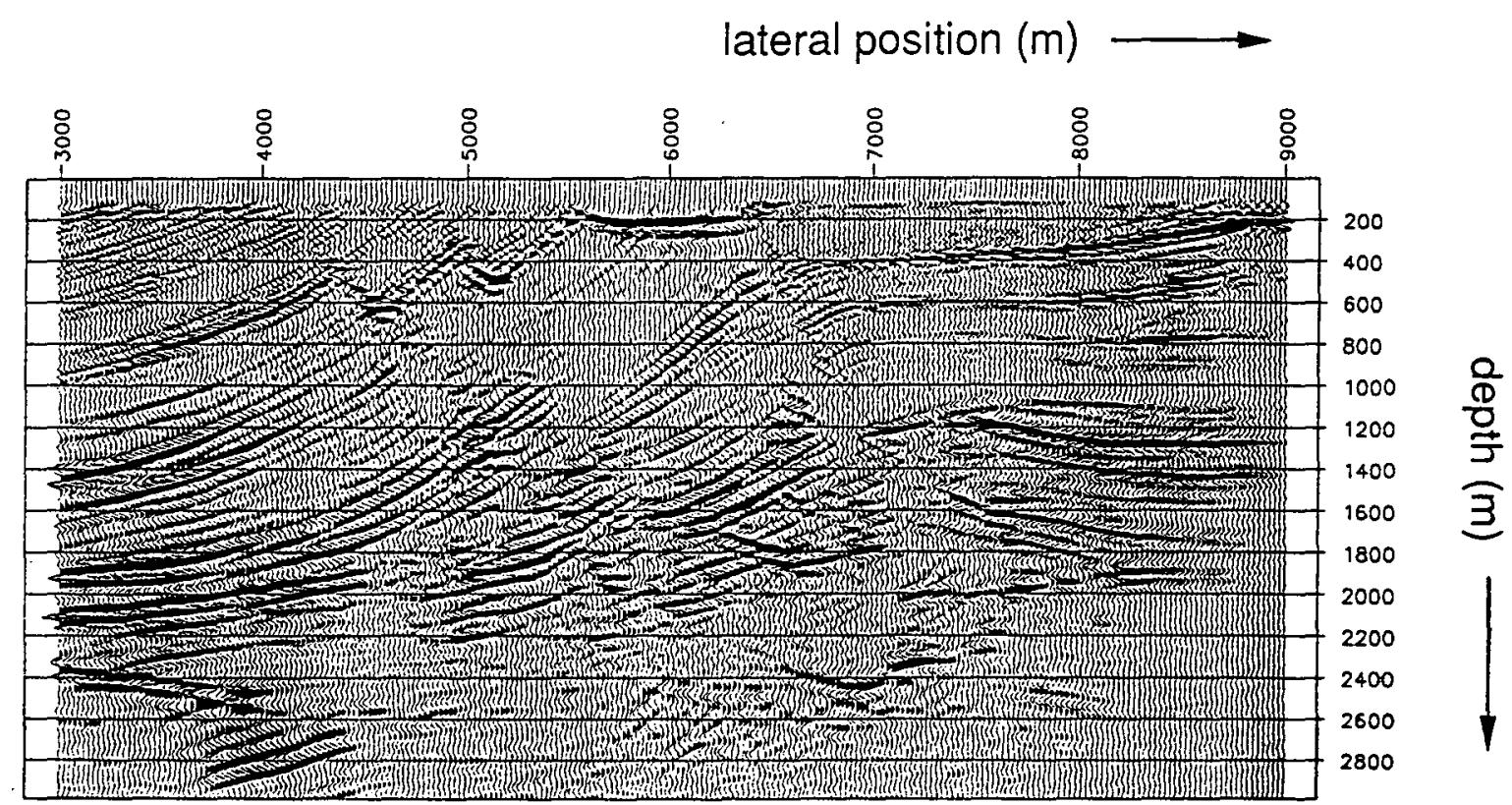

b)

Figure 28

a. Macro model after focussing analysis (first update).

b. Prestack migration result of the preprocessed data. 


\section{Acknowledgment}

The authors would like to express their gratitude to Dr. Gerrit Blacquière of Delft Geophysical B.V., who estimated the initial model from conventional processing and performed the shot record extrapolation for the successive models.

\section{References}

Berkhout, A.J., 1985, Seismic migration: Imaging of acoustic energy by wave field extrapolation. A. Theoretical aspects, third edition: Elsevier Science Publ. Co., Inc.

Berryhill, J.R., and Kim, Y.C., 1986, Deep-water peglegs and multiples: emulation and suppression, Geophysics, Volume 51, 2177 2184.

Cox, H.L.H., 1991, Macro model estimation by wave field extrapolation, D.Sc. thesis, Delft, The Netherlands

Geertsma, J., 1961, Velocity-Log interpretation: The effect of rock bulk compressibility: J.

Soc. Petr. Eng., 235 - 248.

$$
\text { sea }
$$

Jeannot, J.P., Faye, J.P., and Denelle, E.,1986, Prestack migration velocities from focusing depth analysis: 56th SEG meeting, Houston, Expanded Abstracts, 438-440

Kennett, B.L.N., 1979, The suppression of surface multiples on seismic records, Geophysical prospecting 27, 584-600.

Kinneging, N.A., Budejicky, V., Wapenaar, C.P.A., and Berkhout, A.J., 1989, Efficient 2D and 3D Shot Record Redatuming: Geophysical Prospecting 37, 493-530

MacBain, J., 1989, Interval Velocity Analysis for Prestack Depth Migration: 59th SEG meeting, Dallas, Expanded Abstracts, 1233-1237

Verschuur, D. J., Berkhout, A. J., and Wapenaar, C. P. A., 1990, Surface related multiple elimination in the presence of near surface anomalies: 60th Ann. Internat. Mtg., Soc. Expl. Geophys., Expanded Abstracts, 16221625.

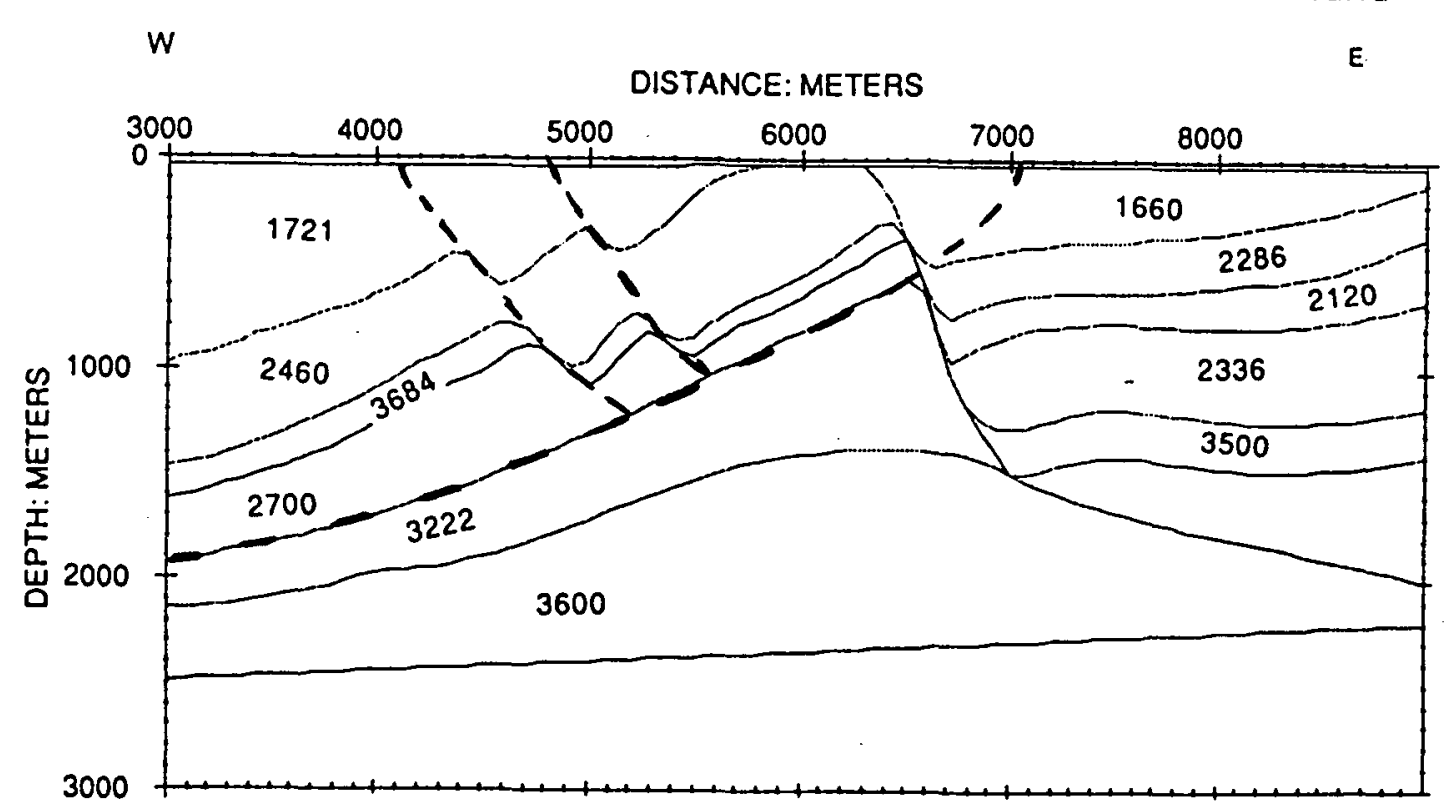

Figure 29

Macro model after focussing analysis and incorporation of the incorrect geologic information. The model contains small counter-regional faults that terminate against a large growth fault. 


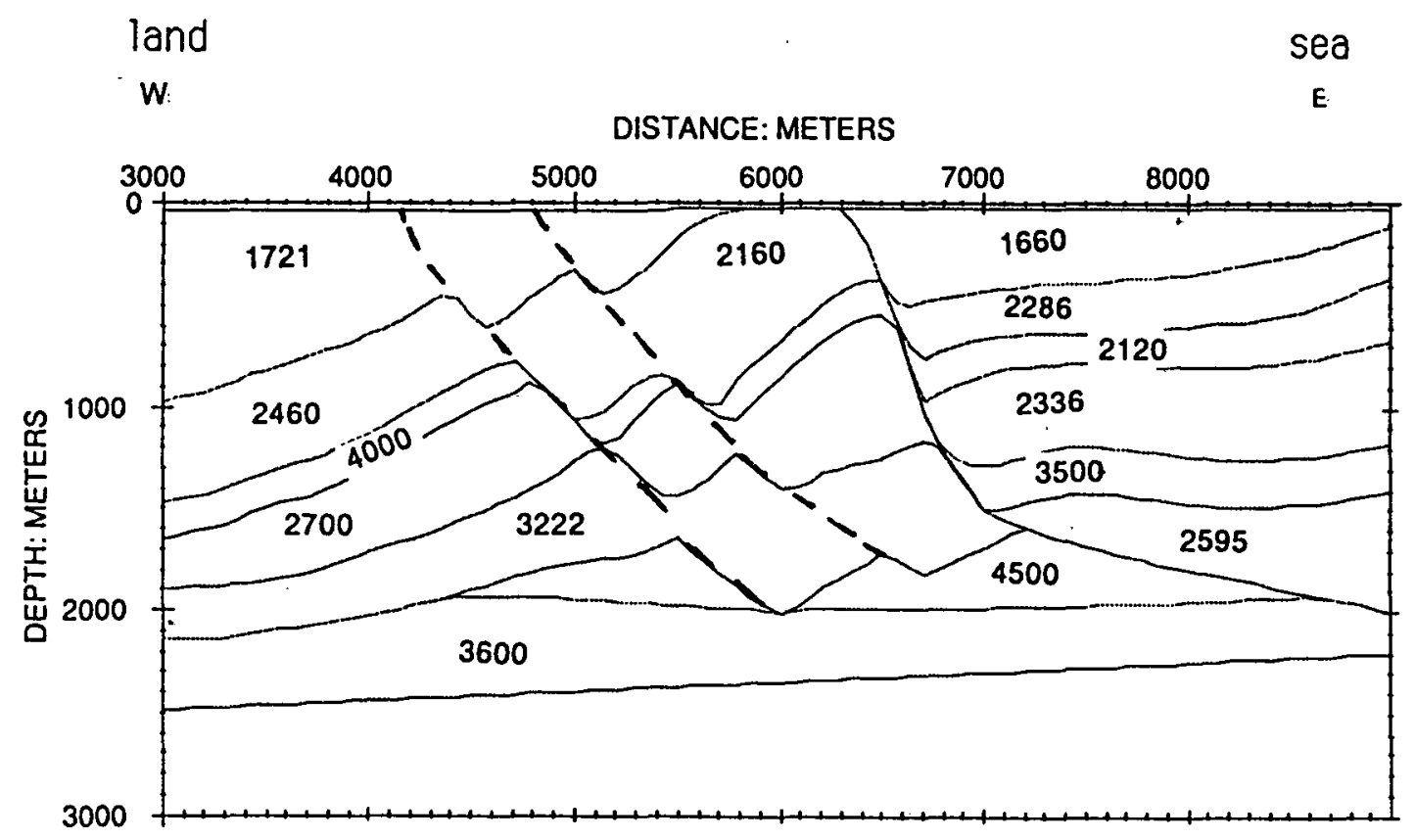

a)

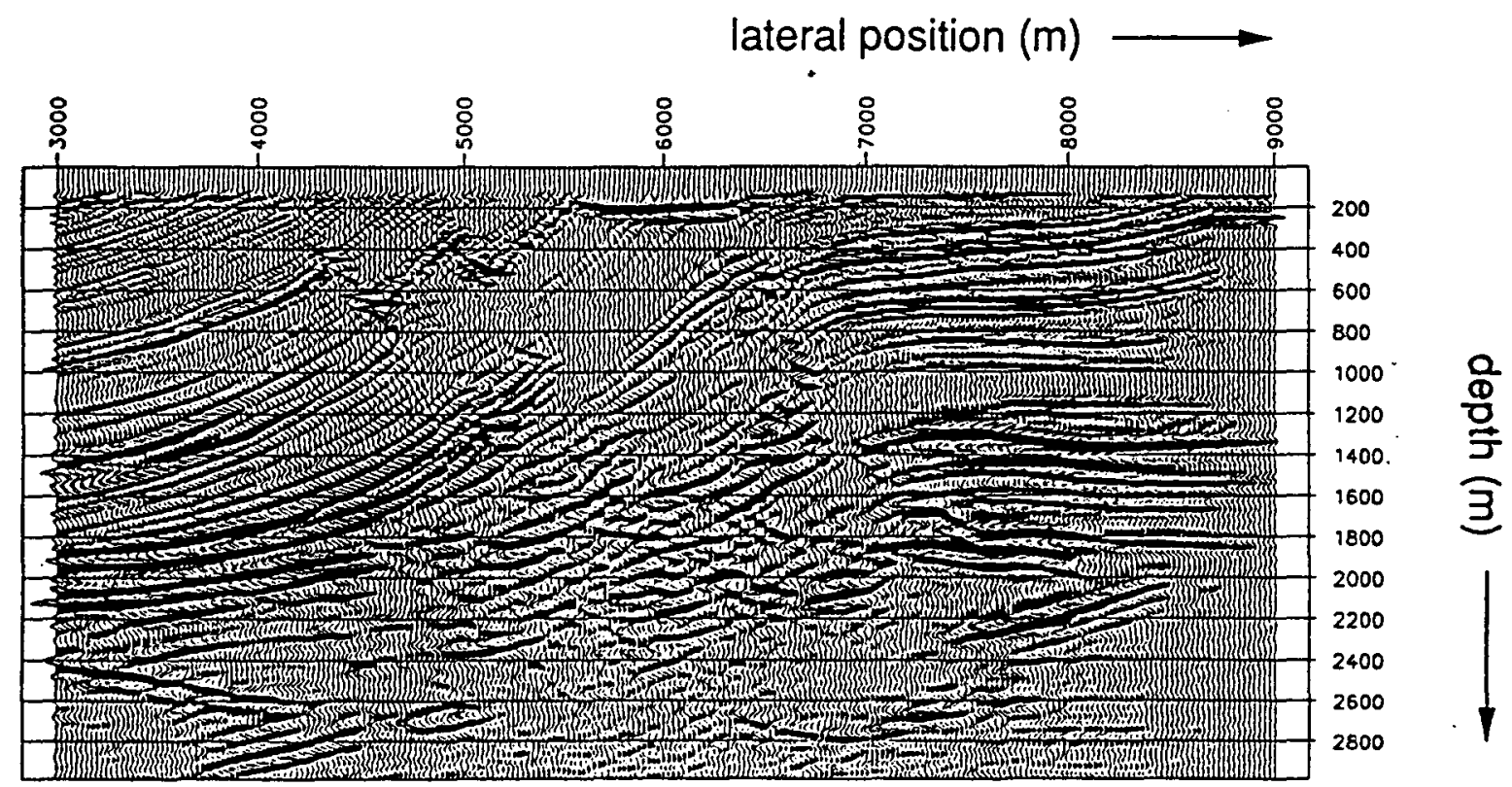

b)

Figure 30

a. Macro model after reinterpretation by incorporating the correct geologic information (see also Colour figure 10).

The model contains three large growth faults.

b. Prestack migration result of the preprocessed data. 


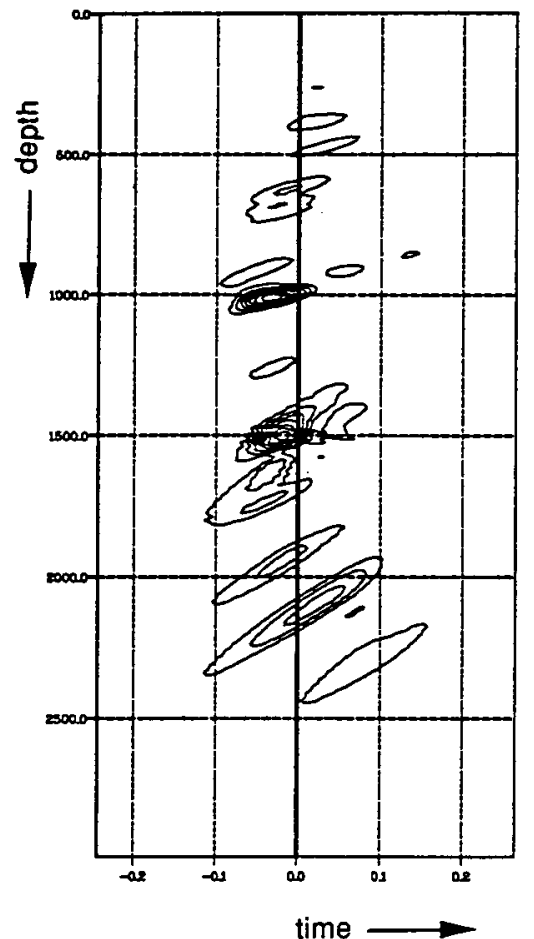

a)

Figure 31

Focus panels at $x=3000 \mathrm{~m}$. (a), $x=5000 \mathrm{~m}$. (b) and $x=7000 \mathrm{~m}$. (c), obtained by shot record extrapolation with the model of Figure 30 a.

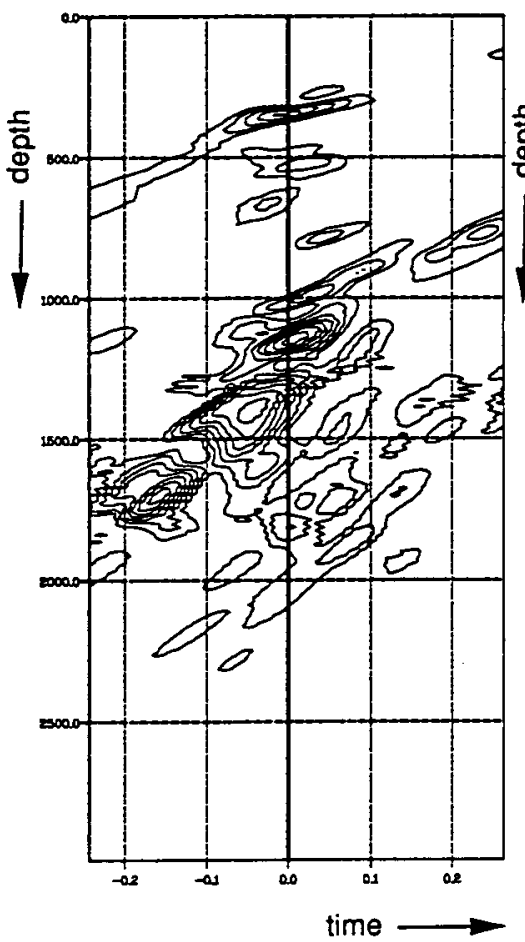

b)

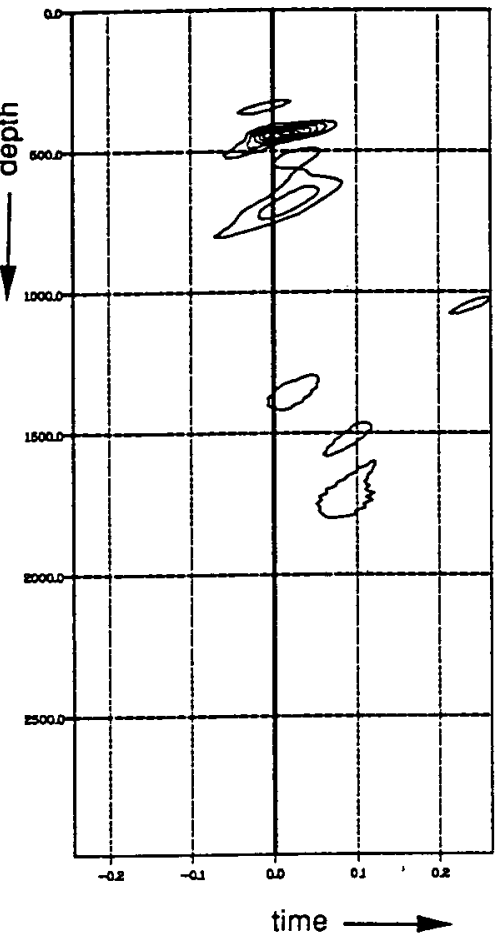

c) 
